\title{
OPTIMAL CONSUMPTION FROM INVESTMENT AND RANDOM ENDOWMENT IN INCOMPLETE SEMIMARTINGALE MARKETS
}

\author{
BY IOANNIS KARATZAS ${ }^{1}$ AND GORDAN ŽITKOVIĆ \\ Columbia University
}

\begin{abstract}
We consider the problem of maximizing expected utility from consumption in a constrained incomplete semimartingale market with a random endowment process, and establish a general existence and uniqueness result using techniques from convex duality. The notion of "asymptotic elasticity" of Kramkov and Schachermayer is extended to the time-dependent case. By imposing no smoothness requirements on the utility function in the temporal argument, we can treat both pure consumption and combined consumptionterminal wealth problems in a common framework. To make the duality approach possible, we provide a detailed characterization of the enlarged dual domain which is reminiscent of the enlargement of $\mathbb{L}^{1}$ to its topological bidual $\left(\mathbb{L}^{\infty}\right)^{*}$, a space of finitely additive measures. As an application, we treat a constrained Itô process market model, as well as a "totally incomplete" model.
\end{abstract}

1. Introduction. Both modern and classical theories of economic behavior use utility functions to describe the amount of "satisfaction" of financial agents from wealth or from consumption. Starting with an initial endowment, an agent is faced with the problem of distributing wealth among financial assets with different degrees of uncertainty. If the market is arbitrage-free, the agent can never "beat the market," but may still invest in such a way as to maximize expected utility. A considerable body of literature has been devoted to this subject. The utility maximization problem in continuous-time stochastic financial market models was first considered by Merton [28, 29], who used a strong assumption (usually not justified in practice) that stock prices are governed by Markovian dynamics with constant coefficients. In this way Merton could use the methods of stochastic dynamic programming and, in particular, the Hamilton-Jacobi-Bellman equation. More recently, a "martingale" approach to the problem in complete Itô process markets was introduced by Pliska [30], Karatzas, Lehoczky and Shreve [22] and Cox and Huang $[4,5]$. They related the marginal utility from the terminal wealth of the optimal portfolio to the density of the (unique) martingale measure using powerful convex-duality techniques.

Difficulties with this approach arise in incomplete markets. The main idea here is to use the convex nature of the problem to formulate and solve a dual variational

Received August 2001; revised July 2002.

${ }^{1}$ Supported by NSF Grant DMS-00-99690.

AMS 2000 subject classifications. Primary 91B28, 91B70; secondary 60G07, 60G44.

Key words and phrases. Utility maximization, random endowment, incomplete markets, convex duality, stochastic processes, finitely additive measures. 
problem and then proceed as in the complete case. In discrete time and on a finite probability space, the problem was studied by He and Pearson [17] and in a continuous-time model was studied by $\mathrm{Xu}$ [38] in his doctoral dissertation, by Karatzas, Lehoczky, Shreve and Xu [23] and by He and Pearson [18]. Kramkov and Schachermayer [26] solved the problem in the context of a general incomplete semimartingale financial market. They showed that a necessary and sufficient condition for the existence of an optimal solution is the so-called reasonable asymptotic elasticity of the utility function at infinity. These authors also showed that the set of densities of local martingale measures is too small to host the solutions of the dual problem. Thus, they enlarged it to a suitably chosen set $y^{*}$ of supermartingales, in a manner reminiscent of enlarging $\mathbb{L}^{1}$ to its topological bidual $\left(\mathbb{L}^{\infty}\right)^{*}$. Although these supermartingales cannot be used directly as pricing rules for derivative securities, Kramkov and Schachermayer showed that this is possible under an appropriate change of numeraire.

When, in addition to initial wealth, the agent faces an uncertain random intertemporal endowment, the situation becomes technically much more demanding and the gap between complete and incomplete markets becomes even more apparent. In the complete market setting the entire uncertain endowment can be "hedged away" in the market, and the problem becomes equivalent to the one where the entire endowment process is replaced by its present value, in the form of an augmented initial wealth. A self-contained treatment of this situation in Itô process models for financial markets can be found in Section 4.4 of the monograph Karatzas and Shreve [21]. An otherwise complete market with random endowment, where the incompleteness is introduced through prohibition of borrowing against future income, is studied in [14].

In incomplete markets, several authors considered this problem in various degrees of generality. We mention Cuoco [6], who dealt with a cone-constrained Itô process market with random endowment. He attacked directly the primal problem circumventing the duality approach altogether, at the cost of rather strict restrictions on the utility function. A definitive solution to the problem of maximizing utility from terminal wealth in incomplete (though not constrained in a more general way) semimartingale markets with random endowment was offered in [8]. The main contribution of that article is the introduction of finitely additive measures into the realm of optimal stochastic control problems encountered in mathematical finance. The essential difference between utility maximization with and without random endowment is probably best described in [8]:

it was not important in the analysis of [26] where the "singular mass of $\hat{\mathbb{Q}}$ has disappeared to." In the present paper this becomes very important ... [it] acts on the accumulated random endowment and can be located in $\left(\mathbb{L}^{\infty}\right)^{*}$.

We finally mention [33] as an extensive survey of the optimal investment theory.

This article advances the existing results in several ways. First, we incorporate intertemporal consumption in the optimization problem. We are dealing with an 
agent who invests in an incomplete market, where prices are modelled by an arbitrary semimartingale with right-continuous and left-limited paths. From the present moment to some finite time horizon $T$, our agent is not only deciding how to manage a portfolio by dynamically readjusting the positions in various financial assets, but also choosing a portion of wealth to be consumed and not further reinvested. The agent also has to take into account the uncertainty in the random endowment stream. It is from this consumption, or from consumption and terminal wealth, that utility is derived. We allow the utility function to be random, reflecting the changes in the agent's risk preferences from one time to another. In a departure from existing theory, we do not impose any smoothness on the utility function in its temporal argument. As a result, we have a common framework for problems that involve consumption only and for problems that involve both consumption and terminal wealth. In addition to dealing with an inherently incomplete semimartingale market model, we impose convex cone constraints on the investment choices the agent is facing. In this way we can model incompleteness and prohibition of short sales, to name only two.

For utility functions we formulate the concept of asymptotic elasticity and, under an appropriate condition of "reasonable asymptotic elasticity," we establish the existence and uniqueness of optimal consumption-investment strategies. In [26] it was only the terminal value of a dual process that appeared in the analysis: the dual domain $\left\{Y_{T}: Y \in \mathcal{Y}\right\} \subseteq \mathbb{L}_{+}^{0}$ was endowed with the topology of convergence in probability on the space $\mathbb{L}_{+}^{0}$ of nonnegative, measurable functions. The more difficult situation in [8] required the dual domain to be extended to the closure of the set of all equivalent martingale measures in $\left(\mathbb{L}^{\infty}\right)^{*}$ - a space that has elements that are finitely additive set functions. Abusing terminology slightly, we call such set functions finitely additive measures. In our case, we have to mimic the natural correspondence between measures and uniformly integrable martingales in the finitely additive world. It turns out that the right choice consists of a dual domain, inhabited, on the one hand, by finitely additive measures and, on the other hand, by "coupled" supermartingales that correspond to the RadonNikodým derivatives of the regular parts of these measures. We prove rigorously that these supermartingales essentially correspond to the supermartingales in the set $y^{*}$ of (2.9) below, which is defined in [26]. The main tool in this endeavor is the filtered bipolar theorem of Žitković [40].

As applications of our results, we treat two special cases: a constrained Itô process market, where we prove that the optimal dual process is always a local martingale, and a "totally incomplete" market as in [27], where the agent is not allowed to invest in the stock market at all.

We stress that an important motivation behind this work is the role it plays as a necessary step for an offensive on the problem of existence and uniqueness for equilibrium in continuous-time incomplete markets with random endowments, a task we plan to attempt in future research. 
The part of our analysis that deals with duality, and especially the structure of the proof of the main result, is closely based on and inspired by the expositions in [26] and [8]. In Section 2 we set up the market model and present a characterization of admissible consumption strategies. Section 3 displays our main result and the Appendix gives its proof. In Section 4 we give an application of our results through two examples.

\section{The model.}

2.1. The financial market. We introduce a model for a financial market consisting of the following:

1. A positive, adapted process $B=\left(B_{t}\right)_{t \in[0, T]}$ with paths that are RCLL (right continuous on $[0, T)$, with left limits everywhere on $(0, T])$ and uniformly bounded from above and away from zero. We interpret $B$ as the numeraire asset—a bond, for example.

2. A RCLL semimartingale $S=\left(S_{t}\right)_{t \in[0, T]}$ taking values in $\mathbb{R}^{d}$; its component processes represent the prices of $d$ risky assets, discounted in terms of the numeraire $B$.

All processes are defined on a filtered probability space $\left(\Omega, \mathcal{F},\left(\mathcal{F}_{t}\right)_{t \in[0, T]}, \mathbb{P}\right)$ with a finite time horizon $T>0$, and the filtration $\mathbb{F} \triangleq\left(\mathcal{F}_{t}\right)_{t \in[0, T]}$ satisfies the "usual conditions" of right continuity and augmentation by $\mathbb{P}$-negligible sets; $\mathcal{F}_{0}$ is the completion of the trivial $\sigma$-algebra.

We consider a financial agent endowed with initial wealth $x>0$ and a random cumulative endowment process $\mathcal{E}=\left(\mathcal{E}_{t}\right)_{t \in[0, T]}$; here $\mathcal{E}_{t}$ is the total (cumulative) amount of endowment received by time $t$. We assume that $\varepsilon_{0}=0$ and $\varepsilon$ is nondecreasing, $\mathbb{F}$-adapted, RCLL and uniformly bounded from above [i.e., $\mathcal{E}_{T} \in$ $\left.\mathbb{L}_{+}^{\infty}(\mathbb{P})\right]$. Similarly to the price process $S$, we assume that $\mathscr{E}$ is already discounted (denominated in terms of $B$ ). Faced with inherent uncertainty about future endowment, the agent dynamically adjusts positions in different financial assets and designates a part of wealth for immediate consumption, in the following manner:

(a) The agent chooses an $S$-integrable and $\mathbb{F}$-predictable process $H$ taking values in $\mathbb{R}^{d}$. The process $H$ has a natural interpretation as a portfolio process; in other words, the $i$ th component of the random vector $H_{t}$ is the number of shares of stock $i$ held at time $t$. To exclude pathologies such as doubling schemes, we choose to impose the condition of admissibility on the agent's choice of portfolio process $H$ by requiring that the gains process $t \mapsto \int_{0}^{t} H_{u}^{\prime} d S_{u}$ be uniformly bounded from below by some real constant. (For the theory of stochastic integration with respect to RCLL semimartingales and the related notions of integrability, consult [31].) Moreover, we ask our agent to obey the investment restrictions imposed on the structure of the market by choosing the portfolio 
process $H$ in a closed, convex cone $\mathcal{K} \subseteq \mathbb{R}^{d}$. The set $\mathcal{K}$ represents constraints on portfolio choice; it can be used to model, for example, short-sale constraints ( $\mathcal{K}=$ $\left.[0, \infty)^{d}\right)$ or the unavailability of some stocks for investment in an incomplete market $\left(\mathcal{K}=\mathbb{R}^{n} \times\{0\} \times \cdots \times\{0\}\right.$ for some $\left.n=1, \ldots, d-1\right)$.

(b) Apart from the choice of portfolio process, the agent chooses a nonnegative, nondecreasing and $\mathbb{F}$-adapted RCLL cumulative consumption process $C=\left(C_{t}\right)_{t \in[0, T]}$. The random variable $C_{t}$ represents the total amount (just like $S_{t}$ and $\varepsilon_{t}$, already discounted by $B_{t}$ ) spent on consumption up to and including time $t$.

A pair $(H, C)$ that satisfies (a) and (b) is called an investment-consumption strategy. The wealth of an agent who employs the investment-consumption strategy $(H, C)$ is given by

$$
W_{t}^{H, C} \triangleq x+\varepsilon_{t}+\int_{0}^{t} H_{u}^{\prime} d S_{u}-C_{t}, \quad 0 \leq t \leq T .
$$

If $(H, C)$ is such that the corresponding wealth process of (2.1) satisfies $W_{T}^{H, C} \geq 0$ a.s., we say that $(H, C)$ is an admissible strategy. If, for a consumption process $C$, we can find a portfolio process $H$ such that $(H, C)$ is admissible, we call $C$ an admissible consumption process and say that $C$ can be financed by $x+\mathcal{E}$ and $H$. Let $\mu$ be an admissible measure, that is, a probability measure on $[0, T]$, diffuse on $[0, T)$, such that $\mu([0, t])<1$ for all $t<T$. For such a measure we define the support $\operatorname{supp} \mu$ to be $[0, T]$ if $\mu$ charges $\{T\}$, and $[0, T)$ otherwise.

We mostly are interested in admissible consumption processes $C$ that can be expressed as

$$
C_{t}=\int_{0}^{t} c(u) \mu(d u), \quad 0 \leq t \leq T .
$$

The set of all densities $c(\cdot)$ of such processes is denoted by $\mathcal{A}^{\mu}(x+\mathcal{E})$. We allow for bulk consumption at the terminal time to be able to deal later on with utility from the terminal wealth and/or from consumption in the same framework. The following notation is used:

$$
\begin{array}{r}
X \triangleq\left\{x+\int_{0}^{\cdot} H_{u}^{\prime} d S_{u}: H \text { is predictable and } S\right. \text {-integrable, } \\
H_{t} \in \mathcal{K} \text { a.s. for every } t \in[0, T], x \geq 0, \\
\left.X(\cdot)=x+\int_{0}^{\cdot} H_{u}^{\prime} d S_{u} \text { is nonnegative }\right\} .
\end{array}
$$

REMARK 1. Even though we allow debt to incur before time $T$, the agent must invest in such a way as to be able to post a nonnegative wealth by the end of the trading horizon with certainty. Furthermore, the boundedness of the process $\mathcal{E}=\left(\mathcal{E}_{t}\right)_{t \in[0, T]}$ guarantees that the negative part of the wealth remains bounded by a constant (a weak form of constrained borrowing). 
2.2. The optimization problem. Let us introduce now a preliminary version of the optimization problem and outline its solution. The goal is to find a consumption-rate process $\hat{c}^{x}(\cdot)$, financed by the initial wealth $x$ and the random endowment $\mathcal{E}$, which maximizes the expected utility from consumption-the "average felicity" of an agent who follows the consumption strategy $\hat{c}^{x}(\cdot)$. The expected utility from a consumption-rate process $c(\cdot)$ is given by

$$
\mathbb{E}\left[\int_{0}^{T} U(t, c(t)) \mu(d t)\right]
$$

where $U$ denotes a (random) utility function and $\mu$ denotes a utility measure. We postpone discussion of the definition and regularity properties of $U$ until Section 3 . In this notation,

$$
\hat{c}^{x}(\cdot)=\underset{c \in \mathcal{A}^{\mu}(x+\mathcal{E})}{\arg \max } \mathbb{E}\left[\int_{0}^{T} U(t, c(t)) \mu(d t)\right] .
$$

As is customary in the duality approach to stochastic optimization, we introduce a problem dual to (2.3) by setting

$$
Y^{\hat{\mathbb{Q}}^{y}}=\underset{\mathbb{Q} \in \mathcal{D}}{\arg \min }\left[\mathbb{E} \int_{0}^{T} V\left(t, y Y_{t}^{\mathbb{Q}}\right) \mu(d t)+y\left\langle\mathbb{Q}, \mathcal{E}_{T}\right\rangle\right] .
$$

Here $\mathcal{D}$ denotes the domain for the dual problem; it is the closure of the set of all supermartingale measures for the stock-price process $S$, and its elements are finitely additive measures. The process $Y^{\mathbb{Q}}$ is a supermartingale version for the density process of $\mathbb{Q}^{r}$, the regular part of $\mathbb{Q}$, and $V$ is the convex conjugate of $U$.

In the following subsection, we describe the dual domain $\mathscr{D}$ in detail and establish some of its properties - the prominent one being weak * compactness. It is precisely this property that ensures the existence of a solution to the dual problem and then, through standard tools of convex duality, ensures the existence of an optimal consumption process $\hat{c}^{x}(\cdot)$ for any initial wealth $x>0$.

2.3. Connections with stochastic control theory. The portfolio process $H$ serves as the analogue of the control process in stochastic control theory. It is important, though, to stress that we are not dealing here with a partially (incompletely) observed problem (a terminology borrowed again from control theory). Incomplete markets in mathematical finance correspond to a setting in which the controller has full information about many aspects of the system (the market), but various exogenously imposed constraints (taxation, transaction costs, bad credit rating, legislation, etc.) prevent him or her from choosing the control (portfolio) outside a given constraint set. In fact, even without governmentimposed portfolio constraints, financial markets typically do not offer tradeable assets that correspond to a variety of sources of uncertainty (weather conditions, nonlisted companies, etc.) The financial agent will still observe many of these 
sources, as their uncertainty evolves, but will typically not be able to "trade in all of them," as it were.

This fundamental nature of financial markets is reflected in our modelling: in Sections 1-3, we allow the filtration $\mathbb{F}$ (with respect to which the controls are adapted) to be possibly larger than the filtration generated by the stock-price process $S$. The only requirement we impose in the next subsection is absence of arbitrage, the fulfilment of which depends heavily on the choice of filtration $\mathbb{F}$. To sum up, the observables in financial modelling constitute a much larger class than the mere stocks in which we are allowed to invest. With such an understanding, our portfolios are adapted only to the observables of the system. Such a setting corresponds to the well-established control-theoretic notion of admitting "open loop" controls in our analysis.

In the more specialized setup of Section 4 , the filtration $\mathbb{F}$ is taken as the augmentation of the filtration generated by the Brownian motions driving the stock prices, assuming as we do in the beginning of Section 4.1 that the volatility matrix process $\sigma(t)$ is nonsingular a.s. for each $t$. At the level of generality considered in this article, the filtration that corresponds to the stock prices will be smaller than the filtration generated by the Brownian motion. However, the two filtrations are actually the same, when interest rates, volatilities and appreciation rates are functions of past and present stock prices; this includes the case of Markovian or deterministic coefficients. In this case, "open loop" and "closed loop" (i.e., $S$-adapted) controls actually coincide.

Finally, we stress that market incompleteness is the main source of technical and conceptual problems we had to overcome in this work, whereas the case of complete markets has been well studied by many authors before; see, for instance, Chapters 3 and 4 in [21]. All of our results that concern the structure of the dual domain (as well as the introduction of the dual domain in the first place) are consequences of the incompleteness of the market. We actually allow for two separate sources of incompleteness-the general structure of the stock prices, as well as the portfolio constraints in the form of the cone $\mathcal{K}$. By choosing $\mathcal{K}=\mathbb{R}^{n} \times\{0\} \times \cdots \times\{0\}$ for some $n=1, \ldots, d-1$, we capture exactly the setting of an incomplete market with $n$ stocks and with $d>n$ sources of randomness that affect the coefficients in the model.

\subsection{Absence of arbitrage, finitely additive set functions and the dual domain.} To make possible a meaningful mathematical treatment of the optimization problem, we excise arbitrage opportunities by postulating the existence of an equivalent supermartingale measure, that is, a probability measure on $(\Omega, \mathcal{F})$, equivalent to $\mathbb{P}$, under which the elements of the set $\mathcal{X}$ in (2.2) become supermartingales. The set of all equivalent supermartingale probability measures is denoted by $\mathcal{M}$ and we assume throughout that $\mathcal{M} \neq \varnothing$. A detailed treatment of the connections between various notions of arbitrage and the existence of equivalent 
martingale (local martingale, supermartingale) measures, culminating with the fundamental theorem of asset pricing, can be found in [9] and [11].

As was pointed out in [8], the duality treatment of utility maximization requires a nontrivial enlargement of $\mathcal{M}$ : this space turns out to be too small, in terms of closedness and compactness properties, for a treatment of the problem at hand. Accordingly, we define $\mathscr{D}$ to be the $\sigma\left(\left(\mathbb{L}^{\infty}\right)^{*}, \mathbb{L}^{\infty}\right)$-closure of $\mathcal{M}$ in $\left(\mathbb{L}^{\infty}\right)^{*}$ - the topological dual of $\mathbb{L}^{\infty}$-where $\mathcal{M}$ is canonically identified with its embedding into $\left(\mathbb{L}^{\infty}\right)^{*}$. [In the terminology of control theory, we are relaxing the set of controls over which the optimization is to be carried out in the dual problem; see (3.3) below, as well as the text following it.] We denote by $\left(\mathbb{L}^{\infty}\right)_{+}^{*}$ the set of nonnegative elements in $\left(\mathbb{L}^{\infty}\right)^{*}$.

The following proposition collects some properties of the spaces $\left(\mathbb{L}^{\infty}\right)^{*}$, $\left(\mathbb{L}^{\infty}\right)_{+}^{*}$ and $\mathcal{D}$; more information about $\left(\mathbb{L}^{\infty}\right)^{*}$ can be found in [1].

PROPOSITION 2.1. (i) The space $\left(\mathbb{L}^{\infty}\right)^{*}$ consists of finitely additive measures on $\mathcal{F}$, which are finite and assign the value zero to $\mathbb{P}$-null subsets of $\mathcal{F}$.

(ii) Under the canonical pairing $\langle\cdot, \cdot\rangle:\left(\mathbb{L}^{\infty}\right)^{*} \times \mathbb{L}^{\infty} \rightarrow \mathbb{R}$, the relation $\langle\mathbb{Q}, 1\rangle=1$ holds for all $\mathbb{Q} \in \mathcal{D}$. In other words, with the notation $\mathbb{Q}(A) \triangleq\left\langle\mathbb{Q}, \mathbf{1}_{A}\right\rangle$ for $A \in \mathcal{F}$ and $\mathbb{Q} \in\left(\mathbb{L}^{\infty}\right)^{*}$, we have $\mathbb{Q}(\Omega)=1$ for all $\mathbb{Q} \in \mathcal{D}$.

(iii) $\mathcal{D}$ is convex and weak $*\left[\right.$ i.e., $\left.\sigma\left(\left(\mathbb{L}^{\infty}\right)^{*}, \mathbb{L}^{\infty}\right)\right]$-compact.

(iv) Every element $\mathbb{Q}$ of $\left(\mathbb{L}^{\infty}\right)_{+}^{*}$ admits a unique decomposition of the form

$$
\mathbb{Q}=\mathbb{Q}^{r}+\mathbb{Q}^{s} \quad \text { with } \mathbb{Q}^{r}, \mathbb{Q}^{s} \in\left(\mathbb{L}^{\infty}\right)_{+}^{*},
$$

where the regular part $\mathbb{Q}^{r}$ is countably additive (it is the maximal countably additive measure on $\mathcal{F}$ that is dominated by $\mathbb{Q}$ ) and the singular part $\mathbb{Q}^{s}$ is purely finitely additive (i.e., does not dominate any nontrivial countably additive measure).

(v) $\mathbb{Q} \in\left(\mathbb{L}^{\infty}\right)_{+}^{*}$ is purely finitely additive (i.e., $\mathbb{Q}^{r} \equiv 0$ ) if and only if for every $\varepsilon>0$ there exists $A_{\varepsilon} \in \mathcal{F}$ such that $\mathbb{P}\left(A_{\varepsilon}\right)>1-\varepsilon$ and $\mathbb{Q}\left(A_{\varepsilon}\right)=0$.

(vi) Suppose a bounded sequence $\left\{\mathbb{Q}_{n}\right\}_{n \in \mathbb{N}}$ in $\left(\mathbb{L}^{\infty}\right)_{+}^{*}$ is such that $d \mathbb{Q}_{n}^{r} / d \mathbb{P} \rightarrow f$ a.s. for some $f \geq 0$. Then any weak $*$ cluster point $\mathbb{Q}$ of $\left\{\mathbb{Q}_{n}\right\}_{n \in \mathbb{N}}$ satisfies $d \mathbb{Q}^{r} / d \mathbb{P}=f$ a.s., where $\mathbb{Q}^{r}$ denotes the regular part of $\mathbb{Q}$.

(vii) The regular-part operator $\mathbb{Q} \mapsto \mathbb{Q}^{r}$ is additive on $\left(\mathbb{L}^{\infty}\right)_{+}^{*}$.

ProOF. (i) See [1], Corollary 4.7.11.

(ii) Follows from the density of $\mathcal{M}$ in $\mathcal{D}$.

(iii) This is the content of Alaoglu's theorem (see [37], Theorem 2.A.9).

(iv) See Theorem 10.2.1 in [1].

(v) See Lemma A.1 in [8].

(vi) See Proposition A.1 in [8].

(vii) Let $\mathbb{Q}_{1}$ and $\mathbb{Q}_{2}$ be elements of $\left(\mathbb{L}^{\infty}\right)_{+}^{*}$. It is enough to show that $\left(\mathbb{Q}_{1}+\right.$ $\left.\mathbb{Q}_{2}\right)-\left(\mathbb{Q}_{1}^{r}+\mathbb{Q}_{2}^{r}\right)=\mathbb{Q}_{1}^{s}+\mathbb{Q}_{2}^{s}$ is singular. Thanks to $(\mathrm{v})$, for any $\varepsilon>0$ we can 
find sets $A_{\varepsilon}$ and $B_{\varepsilon}$ such that $P\left(A_{\varepsilon}\right)>1-\frac{\varepsilon}{2}, P\left(B_{\varepsilon}\right)>1-\frac{\varepsilon}{2}$ and $\mathbb{Q}_{1}^{s}\left(A_{\varepsilon}\right)=$ $\mathbb{Q}_{2}^{s}\left(B_{\varepsilon}\right)=0$. With $C_{\varepsilon} \triangleq A_{\varepsilon} \cap B_{\varepsilon}$ we have $P\left(C_{\varepsilon}\right)>1-\varepsilon$ and $\left(\mathbb{Q}_{1}^{s}+\mathbb{Q}_{2}^{s}\right)\left(C_{\varepsilon}\right)=0$; this completes the proof, by appeal to $(\mathrm{v})$.

REMARK 2. In light of properties (i) and (ii), we may interpret the elements of $\mathscr{D}$ as finitely additive probability measures on $\mathcal{F}$, weakly absolutely continuous with respect to $\mathbb{P}$. For later use, we extend the pairing notation by setting $\langle\mathbb{Q}, X\rangle \triangleq$ $\lim _{n \rightarrow \infty}\langle\mathbb{Q}, X \wedge n\rangle$ for any $\mathbb{Q} \in\left(\mathbb{L}^{\infty}\right)_{+}^{*}, X \in \mathbb{L}_{+}^{0}$.

Our analysis necessitates associating, to every $\mathbb{Q} \in \mathcal{D}$, a nonnegative, RCLL supermartingale $Y^{\mathbb{Q}}=\left(Y_{t}^{\mathbb{Q}}\right)_{t \in[0, T]}$. For $\mathbb{Q} \in \mathcal{M}$, this process is just the RCLL modification of the martingale $\left(\mathbb{E}\left[\frac{d \mathbb{Q}}{d \mathbb{P}} \mid \mathcal{F}_{t}\right]\right)_{t \in[0, T]}$. For arbitrary $\mathbb{Q} \in\left(\mathbb{L}^{\infty}\right)_{+}^{*}$, the construction of $Y^{\mathbb{Q}}$ is rather delicate [see (2.4) and (2.5)]. To make headway on this issue, we let $\mathbb{Q}^{r}$ denote the regular part of $\mathbb{Q}$ and, for any $\sigma$-algebra $g \subseteq \mathcal{F}$, denote by $\mathbb{Q} \mid g$ the restriction of the set function $\mathbb{Q}$ to $g$. Since the regular-part operator $\mathbb{Q} \mapsto \mathbb{Q}^{r}$ depends nontrivially on the domain of $\mathbb{Q}$, we stress that $(\mathbb{Q} \mid g)^{r}$ stands for a countably additive measure on $g$ and, in general, does not equal $\mathbb{Q}^{r} \mid g$ : the regular part and restriction operations do not commute. In fact, we have the following result:

Proposition 2.2. For any two sub- $\sigma$-algebras $\mathcal{g} \subseteq \mathscr{H}$ of $\mathcal{F}$ and every $\mathbb{Q} \in\left(\mathbb{L}^{\infty}\right)^{*}$, we have $(\mathbb{Q} \mid g)^{r} \geq\left.\left(\left.\mathbb{Q}\right|_{\mathcal{H}}\right)^{r}\right|_{\text {g. }}$ In particular, $(\mathbb{Q} \mid g)^{r} \geq \mathbb{Q}^{r} \mid g$.

ProOF. By Proposition 2.1(iv), $(\mathbb{Q} \mid g)^{r}$ is the maximal countably additive measure on $g$ dominated by $\mathbb{Q}$, so it must dominate $(\mathbb{Q} \mid \mathcal{H})^{r} \mid g$-another countably additive measure on $g$ dominated by $\mathbb{Q}$.

For each $\mathbb{Q} \in \mathscr{D}$, Proposition 2.2 shows that the process

$$
L_{t}^{\mathbb{Q}} \triangleq \frac{d\left(\left.\mathbb{Q}\right|_{\mathcal{F}_{t}}\right)^{r}}{d\left(\left.\mathbb{P}\right|_{\mathcal{F}_{t}}\right)}, \quad t \in[0, T],
$$

is a supermartingale, and Proposition 1.3.14 on page 16 in [20] shows that the "regularization"

$$
Y_{t}^{\mathbb{Q}} \triangleq \liminf _{q \searrow t, q \text { rational }} L_{q}^{\mathbb{Q}}, \quad 0 \leq t<T, \quad Y_{T}^{\mathbb{Q}} \triangleq L_{T}^{\mathbb{Q}}
$$

of $L^{\mathbb{Q}}$ is a supermartingale with RCLL paths. When $\mathbb{Q} \in \mathcal{M}$, the process $Y^{\mathbb{Q}}=$ $\left(Y_{t}^{\mathbb{Q}}\right)_{t \in[0, T]}$ of $(2.5)$ is the RCLL modification of the martingale $\left(\mathbb{E}\left[\frac{d \mathbb{Q}}{d \mathbb{P}} \mid \mathcal{F}_{t}\right]\right)_{t \in[0, T]}$. We define also the two sets

$$
y^{\mathcal{M}} \triangleq\left\{Y^{\mathbb{Q}}: \mathbb{Q} \in \mathcal{M}\right\} \quad \text { and } \quad y^{\mathcal{D}} \triangleq\left\{Y^{\mathbb{Q}}: \mathbb{Q} \in \mathscr{D}\right\} \supseteqq y^{\mathcal{M}} .
$$

The following proposition examines some properties of the elements of $\mathcal{Y}^{\mathcal{D}}$. It shows that the regularization (2.5) of the process $L^{\mathbb{Q}}$ of (2.4) is, in fact, a harmless operation. 
Proposition 2.3. (a) For every $\mathbb{Q} \in \mathscr{D}$, there exists a countable set $K \subset$ $[0, T)$, such that $Y_{t}^{\mathbb{Q}}=L_{t}^{\mathbb{Q}}$ for all $t \in[0, T] \backslash K$ almost surely. In particular, $Y^{\mathbb{Q}}=L^{\mathbb{Q}}(\mu \otimes \mathbb{P})$-a.e., for any admissible measure $\mu$.

(b) For every stopping time $S$, we have $Y_{S}^{\mathbb{Q}} \leq L_{S}^{\mathbb{Q}}$ a.s.

PROOF. (a) Let $K$ be the set of discontinuity points of the decreasing function $t \mapsto \mathbb{E}\left[L_{t}^{\mathbb{Q}}\right]=\left(\mathbb{Q} \mid \mathcal{F}_{t}\right)^{r}(\Omega)$, on $[0, T)$; this set is at most countable. For every $t<T$, Fatou's lemma gives

$$
Y_{t}^{\mathbb{Q}} \leq \liminf _{q \searrow t, q \text { rational }} \mathbb{E}\left[L_{q}^{\mathbb{Q}} \mid \mathcal{F}_{t}\right] \leq L_{t}^{\mathbb{Q}} \quad \text { a.s. }
$$

On the other hand, for any sequence of rationals $\left\{q_{n}\right\}_{n \in \mathbb{N}}$ with $q_{n} \searrow t$, the random sequence $\left\{L_{q_{n}}^{\mathbb{Q}}\right\}_{n \in \mathbb{N}}$ is a backward supermartingale and is bounded in $\mathbb{L}^{1}$, so that $L_{q_{n}}^{\mathbb{Q}} \rightarrow Y_{t}^{\mathbb{Q}}$ both in $\mathbb{L}^{1}$ and a.s., thanks to the backward supermartingale convergence theorem (see [3], Theorem 9.4.7, page 338). For each $t \in[0, T] \backslash K$ we have thus $\mathbb{E}\left[Y_{t}^{\mathbb{Q}}\right]=\mathbb{E}\left[L_{t}^{\mathbb{Q}}\right]$, which, together with (2.7) and the fact that $K$ is at most countable, completes the proof of (a).

(b) For an arbitrary stopping time $S$ and $n \in \mathbb{N}$, we put $S^{n}=\left(2^{-n}\left\lfloor 2^{n} S+\right.\right.$ 1」) $\wedge T$, so that $S \leq S^{n} \leq S+2^{-n}$. Therefore, $\left\{S^{n}\right\}_{n \in \mathbb{N}}$ is a sequence of stopping times with finite range a.s. decreasing to $S$. By the definition (2.5) of $Y^{\mathbb{Q}}$ we have $Y_{S}^{\mathbb{Q}}=\liminf _{n} L_{S^{n}}^{\mathbb{Q}}$. If $\left\{t_{1}^{n}, \ldots, t_{m_{n}}^{n}\right\}$ is the range of $S^{n}$, then for any event $A \in \mathcal{F}_{S}$ $\left(\subseteq \mathcal{F}_{S^{n}}, \forall n \in \mathbb{N}\right)$, we have

$$
\begin{aligned}
\mathbb{E}\left[Y_{S}^{\mathbb{Q}} \mathbf{1}_{A}\right] & =\mathbb{E}\left[\liminf _{n} L_{S^{n}}^{\mathbb{Q}} \cdot \mathbf{1}_{A}\right] \leq \liminf _{n} \mathbb{E}\left[L_{S^{n}}^{\mathbb{Q}} \cdot \mathbf{1}_{A}\right] \\
& =\liminf _{n} \sum_{k=1}^{m_{n}} \mathbb{E}\left[L_{t_{k}^{n}}^{\mathbb{Q}} \cdot \mathbf{1}_{A \cap\left\{S^{n}=t_{k}^{n}\right\}}\right] \\
& =\liminf _{n} \sum_{k=1}^{m_{n}}\left(\mathbb{Q} \mid{\mathcal{F}_{t}^{n}}_{t^{n}}\right)^{r}\left(A \cap\left\{S^{n}=t_{k}^{n}\right\}\right) \\
& \leq \liminf _{n} \sum_{k=1}^{m_{n}}\left|\mathbb{Q}, \mathbf{1}_{A \cap\left\{S^{n}=t_{k}^{n}\right\}}\right\rangle=\left\langle\mathbb{Q}, \mathbf{1}_{A}\right\rangle .
\end{aligned}
$$

Therefore, $Y_{S}^{\mathbb{Q}}$ is the density of a (countably additive) measure dominated by $\mathbb{Q}$ on $\mathcal{F}_{S}$ and we conclude that $Y_{S}^{\mathbb{Q}} \leq\left(d\left(\mathbb{Q}_{\mathcal{F}_{S}}\right)^{r}\right) /\left(\left.d \mathbb{P}\right|_{\mathcal{F}_{S}}\right)=L_{S}^{\mathbb{Q}}$ holds almost surely.

The next results, useful for the duality treatment and interesting in their own right, introduce the notion of Fatou convergence and relate it to the more familiar notion of weak $*$ convergence. Fatou convergence is analogous to a.s. convergence in the context of RCLL processes and was used, for example, in [25], [16] and [12]. 
DEFINITION 2.4. Let $\left\{Y^{(n)}\right\}_{n \in \mathbb{N}}$ be a sequence of nonnegative, $\mathbb{F}$-adapted processes with RCLL paths. We say that $\left\{Y^{(n)}\right\}_{n \in \mathbb{N}}$ Fatou-converges to an $\mathbb{F}$-adapted process $Y$ with RCLL paths if there is a countable, dense subset $\mathcal{T}$ of $[0, T]$, such that

$$
Y_{t}=\liminf _{s \downarrow t, s \in \mathcal{T}}\left(\liminf _{n} Y_{s}^{(n)}\right)=\limsup _{s \downarrow t, s \in \mathcal{T}}\left(\limsup _{n} Y_{s}^{(n)}\right) \quad \text { a.s. }
$$

holds for every $t \in[0, T]$. We interpret (2.8) to mean $Y_{t}=\lim _{n} Y_{t}^{(n)}$ a.s. for $t=T$. A set of nonnegative RCLL supermartingales is called Fatou closed if it is closed with respect to Fatou convergence.

Before stating the next proposition we need a technical result (see Lemma 8 in [40]).

LEMMA 2.5. Let $\left\{Y^{(n)}\right\}_{n \in \mathbb{N}}$ be a sequence of nonnegative RCLL supermartingales that Fatou-converge to a nonnegative RCLL supermartingale $Y$. There is a countable set $K \subseteq[0, T)$ such that $Y_{t}=\liminf _{n} Y_{t}^{(n)}$ for all $t \in[0, T] \backslash K$ almost surely.

Proposition 2.6. Let $\mu$ be a probability measure on $[0, T]$ that is diffuse on $[0, T)$. Suppose that $\left\{\mathbb{Q}^{(n)}\right\}_{n \in \mathbb{N}} \subseteq \mathcal{D}$ has a weak $*$ cluster point $\mathbb{Q}^{*} \in \mathscr{D}$ and that the sequence of supermartingales $\left\{Y^{\mathbb{Q}^{(n)}}\right\}_{n \in \mathbb{N}}$ converges in both $(\mu \otimes \mathbb{P})$-a.e. and the Fatou sense. Then the Fatou limit $Y$ coincides with the $(\mu \otimes \mathbb{P})$ limit up to a.e. equivalence and both are equal to $Y^{\mathbb{Q}^{*}}$.

Proof. The two limits are the same $(\mu \otimes \mathbb{P})$-a.e. by Lemma 2.5. By Proposition 2.3, there exists a sequence $\left\{K_{n}\right\}_{n \in \mathbb{N}}$ of countable subsets of $[0, T)$ and a $\mu$-null set $K^{\prime}$ such that

$$
Y_{t}=\lim _{n} Y_{t}^{\mathbb{Q}^{(n)}}=\lim _{n} L_{t}^{\mathbb{Q}^{(n)}} \quad \text { for all } t \in[0, T] \backslash K
$$

holds almost surely, where $K \triangleq K^{\prime} \cup \bigcup_{n \in \mathbb{N}} K_{n}$. By Proposition 2.1(vi), (2.4) and Proposition 2.3, there is a $\mu$-null set $\hat{K} \supseteqq K$ such that

$$
Y_{t}=Y_{t}^{\mathbb{Q}^{*}}=L_{t}^{\mathbb{Q}^{*}} \quad \text { for all } t \in[0, T] \backslash \hat{K}
$$

holds almost surely. Since $[0, T] \backslash \hat{K}$ is dense in $[0, T]$, the right-continuous processes $Y$ and $Y^{\mathbb{Q}^{*}}$ are indistinguishable.

2.5. On a filtered version of the bipolar theorem. Kramkov and Schachermayer ([26], page 909) defined a set $y^{*}$ of supermartingales, which enlarges for the set of densities of equivalent martingale measures; they then used the elements of $y^{*}$ as "dual variables" for the convex-duality approach to utility maximization 
in incomplete markets. In the setup of [26] there is no endowment after time $t=0$, no portfolio constraint and utility comes from terminal wealth only. In terms of the set $\mathcal{X}$ of stochastic integrals in (2.2), the set $\mathcal{Y}^{*}$ under question is defined as

$$
\begin{aligned}
& y^{*} \triangleq\left\{Y: Y \text { is an adapted nonnegative RCLL process such that } Y_{0} \leq 1\right. \\
& \text { and } \left.\left(X_{t} Y_{t}\right)_{t \in[0, T]} \text { is a supermartingale for each process } X \in X\right\} .
\end{aligned}
$$

Obviously, the elements of $y^{*}$ are supermartingales [ just take $H=0$, thus $X \equiv x$, in (2.2)] and $\mathcal{Y}^{*}$ contains the set $\mathcal{Y}^{\mathcal{M}}$ of (2.6): indeed, from the definition of $\mathcal{M}$ in Section 2.3, the process $X$ of (2.2) is a $\mathbb{Q}$ supermartingale, and thus $X Y^{\mathbb{Q}}$ is a $\mathbb{P}$-supermartingale for every $\mathbb{Q} \in \mathcal{M}$. Except in trivial cases, however, $\mathcal{Y}^{*}$ is a true enlargement of $\mathcal{y}^{\mathcal{M}}$; see (2.10) below. An attempt to study the structure of $\mathcal{Y}^{*}$ was made in [40] by establishing and applying a generalization of the bipolar theorem for subsets of $\mathbb{L}_{+}^{0}$ (see [26]); this is a nonlocally-convex version of the classical bipolar theorem of functional analysis. The generalization comes in the form of the filtered bipolar theorem, the statement and relevant definitions of which we recall now from [40].

DEFINITION 2.7. A set of $\mathcal{y}$ of nonnegative, $\mathbb{F}$-adapted processes with RCLL paths is said to be:

(1) (process) solid if for each $Y \in \mathcal{Y}$ and each nonincreasing $\mathbb{F}$-adapted process $B$ with RCLL paths and $B_{0} \leq 1$, we have $Y B \in \mathcal{Y}$;

(2) fork convex, if for any $s \in(0, T]$, any $h \in \mathbb{L}_{+}^{0}\left(\mathcal{F}_{S}\right)$ with $h \leq 1$ a.s. and any $Y^{(1)}, Y^{(2)}, Y^{(3)} \in \mathcal{Y}$, we also have $Y \in \mathcal{H}$, where

$$
Y_{t} \triangleq \begin{cases}Y_{t}^{(1)}, & 0 \leq t<s, \\ Y_{s}^{(1)}\left(h \frac{Y_{t}^{(2)}}{Y_{s}^{(2)}}+(1-h) \frac{Y_{t}^{(3)}}{Y_{s}^{(3)}}\right), & s \leq t \leq T .\end{cases}
$$

DEFINITION 2.8. Let $y$ be a set of nonnegative, $\mathbb{F}$-adapted processes with RCLL paths. The ( process) polar of $\mathcal{Y}$, is the set $\mathcal{Y}^{\times}$of all nonnegative, $\mathbb{F}$-adapted processes $X$ with RCLL paths, such that $X Y=\left(X_{t} Y_{t}\right)_{t \in[0, T]}$ is a supermartingale with $(X Y)_{0} \leq 1$ for all $Y \in \mathcal{Y}$.

We can now state a mild extension of the main result in [40]. The additional statement (last sentence of Theorem 2.9 below) follows directly from the proof of the original version.

THEOREM 2.9 (Filtered bipolar theorem). Let $y$ be a set of nonnegative and $\mathbb{F}$-adapted processes with RCLL paths, with $Y_{0} \leq 1$ for each $Y \in \mathcal{y}$ and with $Y_{T}>0$ a.s. for at least one $Y \in \mathcal{Y}$. The process bipolar $\mathcal{y}^{\times \times}=\left(\mathcal{y}^{\times}\right)^{\times}$of $\mathcal{y}$ is the smallest Fatou-closed, fork-convex and solid set of $\mathbb{F}$-adapted processes $Y$ with RCLL paths and $Y_{0} \leq 1$ that contains $y$. Furthermore, every element of $\mathcal{y}^{\times \times}$can be obtained as the Fatou limit of a sequence in the solid and fork-convex hull of $\mathcal{y}$. 
2.6. Structural properties of the sets in (2.6) and (2.9). Now let us revisit the sets $\mathcal{y}^{\mathcal{D}}, \mathcal{y}^{\mathcal{M}}$ and $\mathcal{y}^{*}$ of (2.6) and (2.9). From Theorem 4 in [40], we know that the set

$y^{\mathcal{M}}$ of (2.6) is fork convex, and its process bipolar is the set of (2.9):

$$
y^{*}=\left(y^{\mathcal{M}}\right)^{\times \times} \text {. }
$$

It follows from Theorem 2.9 and (2.10) that $\mathcal{y}^{*}$ is the solid and Fatou-closed hull of $y^{\mathcal{M}}$. The task we undertake in this subsection is to formulate and establish formally the statement put forth by the authors in [8], to the effect that

... the idea of passing from $\mathcal{M}$ to $\mathscr{D}$ (introduced in [8]) had already been implicitly present in [26] (disguised in the definition of $y^{*}$ ).

Namely, we shall show that $y^{\mathscr{D}} \subseteq y^{*}$ (in other words, $y^{\mathcal{M}} \subseteq y^{\mathscr{D}} \subseteq y^{*}$ ) and that $y^{D}$ already contains all maximal elements of $\mathcal{y}^{*}$. More precisely, we have the following result.

THEOREM 2.10. The set $\mathcal{Y}^{D}$ in (2.6) is fork convex and Fatou closed, and its solid hull is the set $\mathcal{H}^{*}$ of (2.9).

PROOF. Since $y^{*}$ is the process bipolar of $y^{\mathcal{M}}$ from (2.10) and $\mathcal{y}^{\mathcal{M}}$ is already contained in $\mathcal{Y}^{\mathcal{D}}$, by the filtered bipolar theorem, Theorem 2.9 , it is enough to show that $y^{\mathcal{D}}$ is contained in $y^{*}$, and is fork convex and Fatou closed.

- We prove first the inclusion $y^{\mathcal{D}} \subseteq \mathcal{Y}^{*}$. Let $X \in \mathcal{X}$ be such that $X_{0}=1$ and let $Y \in \mathcal{Y}^{\mathcal{D}}$. By the definition (2.9) of $\mathcal{H}^{*}$, it is enough to show that $X Y$ is a supermartingale and by Proposition 2.3 , it is enough to prove that $X L^{\mathbb{Q}}$ is a supermartingale with $L^{\mathbb{Q}}$ defined in [7]. Equivalently, we have to prove $\left\langle\left(\mathbb{Q} \mid \mathcal{F}_{s}\right)^{r}, X_{s} \mathbf{1}_{A}\right\rangle \geq\left\langle\left(\mathbb{Q} \mid \mathcal{F}_{t}\right)^{r}, X_{t} \mathbf{1}_{A}\right\rangle$ for all $0 \leq s<t \leq T, A \in \mathcal{F}_{s}$ (notation of Remark 2); without loss of generality, we may assume that $X_{S}$ is bounded on $A$.

Recall that for $\mathbb{Q} \in \mathcal{M}$, the process $X$ is a nonnegative $\mathbb{Q}$-supermartingale. By the density of $\mathcal{M}$ in $\mathscr{D}$, we easily conclude that $\left\langle\mathbb{Q}, X_{s} \mathbf{1}_{A}\right\rangle \geq\left\langle\mathbb{Q},\left(X_{t} \wedge m\right) \mathbf{1}_{A}\right\rangle$ holds for every $\mathbb{Q} \in \mathcal{D}$ and $m \in(0, \infty)$ large enough. The singular-part operator is positive, so we have

$$
\left\langle\left(\left.\mathbb{Q}\right|_{\mathcal{F}_{s}}\right)^{r}, X_{s} \mathbf{1}_{A}\right\rangle+\left\langle\left(\left.\mathbb{Q}\right|_{\mathcal{F}_{s}}\right)^{s}, X_{s} \mathbf{1}_{A}\right\rangle \geq\left\langle\left(\left.\mathbb{Q}\right|_{\mathcal{F}_{t}}\right)^{r},\left(X_{t} \wedge m\right) \mathbf{1}_{A}\right\rangle \quad \forall m \in(0, \infty) .
$$

Proposition 2.1(v) guarantees the existence of a sequence of sets $\left\{A_{n}\right\}_{n \in \mathbb{N}}$ in $\mathcal{F}_{s}$ such that $\mathbb{P}\left[A_{n}\right]>1-2^{-n}$ and $\left(\left.\mathbb{Q}\right|_{\mathcal{F}_{s}}\right)^{S}\left(A_{n}\right)=0$. We get

$$
\left\langle\left(\left.\mathbb{Q}\right|_{\mathcal{F}_{s}}\right)^{r} X_{s} \mathbf{1}_{A \cap A_{n}}\right\rangle \geq\left\langle\left(\left.\mathbb{Q}\right|_{\mathcal{F}_{t}}\right)^{r}\left(X_{t} \wedge m\right) \mathbf{1}_{A \cap A_{n}}\right\rangle \quad \forall m \in(0, \infty), n \in \mathbb{N},
$$

and the claim follows by letting $m, n \rightarrow \infty$.

- The fork-convexity of $\mathcal{Y}^{\mathcal{D}}$ follows from the fork-convexity of $y^{\mathcal{M}}$ and from the fact (Theorem 2.9) that every $Y \in \mathcal{Y}^{\mathcal{D}} \subseteq \mathcal{Y}^{*}$ can be Fatou-approximated by a sequence in $\mathcal{y}^{\mathcal{M}}$. 
- As for Fatou-closedness, we take a sequence $\left\{Y^{(n)}\right\}_{n \in \mathbb{N}} \subseteq y^{\mathcal{D}}$ that is Fatouconverging toward a supermartingale $Y$. Let $\lambda$ stand for normalized Lebesgue measure on $[0, T]$. By Komlós' theorem (see [24, 34]) and the convexity of $\mathcal{Y}^{\mathcal{D}}$, we can assume that $\left\{Y^{(n)}\right\}_{n \in \mathbb{N}}$ converges to $(\lambda \otimes \mathbb{P})$-a.e. by passing if necessary to a sequence of convex combinations (note that this operation preserves the Fatou limit). Let $\left\{\mathbb{Q}^{n}\right\}_{n \in \mathbb{N}} \subseteq \mathcal{D}$ be a sequence such that $Y^{(n)}=Y^{\mathbb{Q}^{n}}$. By the weak $*$ compactness of $\mathscr{D}$ from Proposition 2.1(iii), the sequence $\left\{\mathbb{Q}^{n}\right\}_{n \in \mathbb{N}}$ possesses a cluster point $\mathbb{Q}^{*}$. Proposition 2.6 now yields $Y=Y^{\mathbb{Q}^{*}}$, implying the Fatou-closedness of $\mathcal{y}^{\mathscr{D}}$.

For future use, we restate the result of Theorem 2.10 in the following terms.

COROLlaRY 2.11. Every $Y \in \mathcal{y}^{*}$ can be written as $Y=Y^{\mathbb{Q}} B$ for some $\mathbb{Q} \in \mathcal{D}$ in the notation of $(2.5)$, where $B$ is a nonincreasing, $\mathbb{F}$-adapted process with $0 \leq B_{T} \leq B_{0} \leq 1$ and RCLL paths. The process $Y^{\mathbb{Q}} \in \mathcal{Y}^{D}$ can be obtained as the Fatou limit of a sequence of martingales in $y^{\mathcal{M}}$.

Let us also state the following consequence of Theorem 2.10, which is of some independent probabilistic interest; it establishes a one-to-one correspondence between positive supermartingales and finite, positive, finitely additive measures.

COROLlaRY 2.12. Suppose the filtered probability space $(\Omega, \mathcal{F}$, $\left.\left(\mathcal{F}_{t}\right)_{t \in[0, T]}, \mathbb{P}\right)$ satisfies the "usual conditions" and $\mathcal{F}_{0}=\{\varnothing, \Omega\} \bmod \mathbb{P}$. Then every nonnegative local martingale $Y$ with RCLL paths is of the form $Y=Y^{\mathbb{Q}}$ in (2.5) for some nonnegative, finitely additive measure $\mathbb{Q}$.

2.7. A characterization of admissible consumption processes. The enlargement of the dual domain from $\mathcal{M}$ to $\mathcal{D}$ necessitates a reformulation of certain old results in the new setting. As given in Section 2.1, the definition of an admissible consumption process is not very intuitive or useful. To remedy this situation, we establish a budget-constraint characterization of admissible consumption processes, analogous to Theorem 3.6 on page 166 of [21].

PROPOSITION 2.13. A nondecreasing, right-continuous and $\mathbb{F}$-adapted process $C$ with $C_{0}=0$ is an admissible cumulative consumption process if and only if

$$
\mathbb{E}\left[\int_{0}^{T} Y_{t}^{\mathbb{Q}} d C_{t}\right] \leq x+\left\langle\mathbb{Q}, \mathcal{E}_{T}\right\rangle \quad \text { for all } \mathbb{Q} \in \mathcal{D}
$$

PROOF. Let $C$ be a nondecreasing, adapted and right-continuous process that satisfies $C_{0}=0$ and (2.11). By the left continuity and the existence of right limits for the process $t \mapsto C_{t-}$, the stochastic integral $M_{t} \triangleq \int_{0}^{t} C_{u-} d Y_{u}^{\mathbb{Q}}, 0 \leq t \leq T$, is 
a local martingale ([31], Theorem III, page 17), so we can find a nondecreasing sequence of stopping times $\left\{T_{n}\right\}_{n \in \mathbb{N}}$ such that the processes $M_{\bullet}^{T_{n}} \equiv M_{\bullet} \wedge T_{n}$ are uniformly integrable martingales for each $n \in \mathbb{N}$ and $\mathbb{P}\left[T_{n}=T\right] \rightarrow 1$ as $n \rightarrow \infty$. By the assumption (2.11) and the integration-by-parts formula, we have

$$
\begin{aligned}
x+\mathbb{E}^{\mathbb{Q}}\left(\mathcal{E}_{T}\right) & =x+\left\langle\mathbb{Q}, \mathcal{E}_{T}\right\rangle \geq \mathbb{E} \int_{0}^{T} Y_{t}^{\mathbb{Q}} d C_{t}=\lim _{n} \mathbb{E} \int_{0}^{T_{n}} Y_{t}^{\mathbb{Q}} d C_{t} \\
& =\lim _{n} \mathbb{E}\left(\int_{0}^{T_{n}} Y_{t-}^{\mathbb{Q}} d C_{t}+\sum_{s \leq T_{n}} \Delta Y_{s}^{\mathbb{Q}} \Delta C_{s}\right) \\
& =\lim _{n} \mathbb{E}\left(Y_{T_{n}}^{\mathbb{Q}} C_{T_{n}}-\int_{0}^{T_{n}} C_{t-} d Y_{t}^{\mathbb{Q}}\right)=\lim _{n} \mathbb{E}^{\mathbb{Q}}\left[C_{T_{n}}\right]=\mathbb{E}^{\mathbb{Q}}\left(C_{T}\right)
\end{aligned}
$$

for every $\mathbb{Q} \in \mathcal{M}$. Let us define

$$
Z_{t} \triangleq \underset{\mathbb{Q} \in \mathcal{M}}{\operatorname{ess} \sup } \mathbb{E}^{\mathbb{Q}}\left[C_{T}-\mathcal{E}_{T} \mid \mathcal{F}_{t}\right], \quad 0 \leq t \leq T .
$$

From Theorem 2.1.1 in [15], the process $Z$ is a supermartingale under each $\mathbb{Q} \in \mathcal{M}$, with a RCLL modification. Choose this RCLL version for $Z$. Moreover, $Z$ is uniformly bounded from below and $Z_{0} \leq x$; this is because $\mathbb{E}^{\mathbb{Q}}\left[C_{T}-\varepsilon_{T}\right] \leq x$ for every $\mathbb{Q} \in \mathcal{M}$, thanks to (2.12). Applying the constrained version of the optional decomposition theorem (see [16], Theorem 4.1) to $Z$, we can assert the existence of an admissible portfolio $\hat{H}$ and of a nondecreasing optional process $F$ with $F_{0}=x-Z_{0} \geq 0$, such that $Z_{t}=\hat{X}_{t}-F_{t}$, where $\hat{X}_{t} \triangleq x+\int_{0}^{t} \hat{H}_{u}^{\prime} d S_{u}$. On the other hand, by the increase of $C$ we have

$$
\hat{X}_{t}-F_{t}=Z_{t} \geq C_{t}-\underset{\mathbb{Q} \in \mathcal{M}}{\operatorname{essinf}} \mathbb{E}^{\mathbb{Q}}\left[\mathcal{E}_{T} \mid \mathcal{F}_{t}\right], \quad t \in[0, T]
$$

so that in the notation of (2.1) we have $W_{T}^{\hat{H}, C}=\hat{X}_{T}-C_{T}+\mathcal{E}_{T} \geq F_{T} \geq F_{0} \geq 0$ a.s.; this implies the admissibility of the strategy $(\hat{H}, C)$.

Conversely, let $C$ be an admissible consumption process. There exists then an admissible portfolio process $H$ such that the process $X . \triangleq x+\int_{0}^{*} H_{u}^{\prime} d S_{u}$ satisfies $X_{T}-C_{T}+\mathcal{E}_{T} \geq 0$. By the supermartingale property of $X$ under every $\mathbb{Q} \in \mathcal{M}$, we see that $x=\mathbb{E}^{\mathbb{Q}}\left(X_{0}\right) \geq \mathbb{E}^{\mathbb{Q}}\left(X_{T}\right) \geq \mathbb{E}^{\mathbb{Q}}\left(C_{T}\right)-\mathbb{E}^{\mathbb{Q}}\left(\mathcal{E}_{T}\right)$ and conclude $\left\langle\mathbb{Q}, C_{T}\right\rangle \leq x+\left\langle\mathbb{Q}, \mathcal{E}_{T}\right\rangle \forall \mathbb{Q} \in \mathcal{M}$.

To go from this inequality to (2.11), suppose first that $C$ is uniformly bounded from above by a constant $M$ and define its right-continuous inverse

$$
D_{s} \triangleq \inf \left\{t \geq 0: C_{t}>s\right\} \in[0, \infty] \quad \text { for } 0 \leq s<\infty .
$$

For arbitrary but fixed $\mathbb{Q} \in \mathscr{D}$, Theorem 55 in [13] and Fubini's theorem give

$$
\begin{aligned}
& \mathbb{E}\left[\int_{0}^{T} Y_{t}^{\mathbb{Q}} d C_{t}\right]=\mathbb{E}\left[\int_{0}^{M} Y_{D_{s}}^{\mathbb{Q}} \mathbf{1}_{\left\{D_{s}<\infty\right\}} d s\right]=\int_{0}^{M} \phi(s) d s \\
& \text { where } \phi(s):=\mathbb{E}\left[Y_{D_{s}}^{\mathbb{Q}} \mathbf{1}_{\left\{D_{s}<\infty\right\}}\right] .
\end{aligned}
$$


By the supermartingale property of $Y^{\mathbb{Q}}$ and the increase of $D$, the function $\phi(\cdot)$ is nonincreasing, so we can find a countable set $K$, dense in $[0, M]$, that contains all discontinuity points of $\phi(\cdot)$. For an enumeration $\left\{s_{k}\right\}_{k \in \mathbb{N}}$ of $K$, the topology on $\mathscr{D}$ induced by

$$
\left.d\left(\mathbb{Q}_{1}, \mathbb{Q}_{2}\right)=\left|\left\langle\mathbb{Q}_{1}-\mathbb{Q}_{2}, C_{T}\right\rangle\right|+\sum_{k} 2^{-n}|| \mathbb{Q}_{1}-\mathbb{Q}_{2}, \mathbf{1}_{\left\{D_{s_{k}}<\infty\right\}}\right\rangle \mid
$$

is coarser than the weak $*$ topology on $\mathscr{D}$, so we can find a sequence $\left\{\mathbb{Q}^{n}\right\}_{n \in \mathbb{N}} \subseteq \mathcal{M}$ such that

$$
\left\langle\mathbb{Q}^{n}, C_{T}\right\rangle \rightarrow\left\langle\mathbb{Q}, C_{T}\right\rangle \quad \text { and } \quad\left\langle\mathbb{Q}^{n}, \mathbf{1}_{\left\{D_{s}<\infty\right\}}\right\rangle \rightarrow\left\langle\mathbb{Q}, \mathbf{1}_{\left\{D_{s}<\infty\right\}}\right\rangle \quad \text { as } n \rightarrow \infty
$$

for every $s \in K$. Such choice for the sequence $\left\{\mathbb{Q}^{n}\right\}_{n \in \mathbb{N}}$ implies that $\phi^{n}(s)=$ $\mathbb{E}^{\mathbb{Q}^{n}}\left[\mathbf{1}_{\left\{D_{s}<\infty\right\}}\right]$ converges to $\left\langle\mathbb{Q}, \mathbf{1}_{\left\{D_{s}<\infty\right\}}\right\rangle$ for every $s \in K$. Using again Theorem 55 in [13], the integration-by-parts formula from the first part of the proof and the dominated convergence theorem, we get

$$
\begin{aligned}
x+\left\langle\mathbb{Q}, \mathcal{E}_{T}\right\rangle & =x+\lim _{n}\left\langle\mathbb{Q}^{n}, \boldsymbol{E}_{T}\right\rangle \geq \lim _{n}\left\langle\mathbb{Q}^{n}, C_{T}\right\rangle=\lim _{n} \mathbb{E}\left[\int_{0}^{T} Y_{t}^{\mathbb{Q}^{n}} d C_{t}\right] \\
& =\lim _{n} \int_{0}^{M} \phi^{n}(s)=\int_{0}^{M}\left\langle\mathbb{Q}, \mathbf{1}_{\left\{D_{s}<\infty\right\}}\right\rangle d s .
\end{aligned}
$$

Whereas $D_{s}$ is a stopping time, Proposition 2.3(b) yields

$$
\begin{aligned}
\int_{0}^{M}\left\langle\mathbb{Q}, \mathbf{1}_{\left\{D_{s}<\infty\right\}}\right\rangle d s & \geq \int_{0}^{M} \mathbb{E}\left[\frac{d\left(\left.\mathbb{Q}\right|_{\mathcal{F}_{D_{s}}}\right)^{r}}{d\left(\left.\mathbb{P}\right|_{\mathcal{F}_{D_{s}}}\right)} \mathbf{1}_{\left\{D_{s}<\infty\right\}}\right] d s \\
& \geq \int_{0}^{M} \mathbb{E}\left[Y_{D_{s}}^{\mathbb{Q}} \mathbf{1}_{\left\{D_{s}<\infty\right\}}\right] d s \\
& =\mathbb{E} \int_{0}^{T} Y_{t}^{\mathbb{Q}} d C_{t},
\end{aligned}
$$

which establishes that (2.11) holds for $C$ bounded.

We turn now to the case of $C$ which is not necessarily bounded. For each $M \in \mathbb{N}$, the truncated consumption process $C^{M}=C \wedge M$ is admissible, and we have just shown that (2.11) holds with $C$ replaced by $C \wedge M$. Passing to the limit as $M \rightarrow \infty$ on the left-hand side of (2.11) is justified by the increase of the trajectories of $C$ and the monotone convergence theorem.

REMARK 3. The reason for the rather lengthy and technical proof of this result (to be more precise, for the authors' inability to find a shorter one) comes from two rather "unpleasant" facts. First, $\left(\mathbb{L}^{\infty}\right)^{*}$ is not metrizable and, second, Fubini's theorem fails in the setting of finitely additive measures; see [39], Theorem 3.3, page 57 , for such a counterexample. 


\section{The optimization problem.}

3.1. The preference structure. Apart from external factors, such as market conditions and the randomness of the endowment process $\mathcal{E}$, it is important to describe the agent's "preference structure" (or idiosyncratic rapport with risk). We adopt the von Neyman-Morgenstern utility approach to risk aversion and proceed to define a utility random field $U:[0, T] \times \Omega \times \mathbb{R}_{+} \rightarrow \mathbb{R}$.

We impose no smoothness conditions in the time parameter. Instead, we control the range of the marginal utility. As seen in [26] (in the setting of an incomplete semimartingale market with initial endowment only and utility from terminal wealth), a condition of reasonable asymptotic elasticity is both necessary and sufficient for the existence of an optimal investment policy. This is the reason for extending the notion of asymptotic elasticity to the time-dependent case and for restricting our analysis to reasonably elastic utilities only.

DEFINITION 3.1. A jointly measurable function $U:[0, T] \times \Omega \times \mathbb{R}_{+} \rightarrow \mathbb{R}$ is called a (reasonably elastic) utility random field if it has the following properties (unless specified otherwise, all these properties are assumed to hold almost surely and the argument $\omega \in \Omega$ is consistently suppressed):

1. For a fixed $t \in[0, T], U(t, \cdot)$ is strictly concave, increasing, of class $C^{1}$ and satisfies the so-called Inada conditions $\partial_{2} U(t, 0+)=\infty$ and $\partial_{2} U(t, \infty)=0$ a.s. In other words, $U(t, \cdot)$ is a utility function.

2. There are continuous, strictly decreasing (nonrandom) functions $K_{1}: \mathbb{R}_{+} \rightarrow$ $\mathbb{R}_{+}$and $K_{2}: \mathbb{R}_{+} \rightarrow \mathbb{R}_{+}$such that for all $t \in[0, T]$ and $x>0$, we have $K_{1}(x) \leq \partial_{2} U(t, x) \leq K_{2}(x)$ and $\lim \sup _{x \rightarrow \infty}\left(K_{2}(x) / K_{1}(x)\right)<\infty$.

3. The function $t \mapsto U(t, 1)$ is uniformly bounded and

$$
\lim _{x \rightarrow \infty}(\underset{t, \omega}{\operatorname{essinf}} U(t, x))>0 .
$$

4. $U$ is reasonably elastic, that is, its asymptotic elasticity satisfies $\operatorname{AE}[U]<1$ a.s., where

$$
\mathrm{AE}[U]:=\limsup _{x \rightarrow \infty}\left(\underset{t, \omega}{\operatorname{ess} \sup } \frac{x \partial_{2} U(t, x)}{U(t, x)}\right) .
$$

5. For every $x>0$, the stochastic process $U(\cdot, x)$ is $\mathbb{F}$-progressively measurable.

REMARK 4. Condition 3 is the least restrictive. In fact, it serves only to simplify the analysis, by excluding some trivial cases and ensuring that the expression $\mathrm{AE}[U]$ of part 4 is well defined. It is an immediate consequence of Conditions 2 and 3 that the function $t \mapsto U\left(x_{0}, t\right)$ is bounded, for every $x_{0}>0$ a.s. Also, the function $U(t, \infty)$ is either bounded or we have $U(t, \infty)=\infty$ for all $t$ a.s. 
EXAMPLE 3.2. Let $\hat{U}: \mathbb{R}_{+} \rightarrow \mathbb{R}_{+}$be a utility function as in Definition 3.1(1), with $\hat{U}(\infty)>0$ and $\limsup _{x \rightarrow \infty}\left(x \hat{U}^{\prime}(x) / \hat{U}(x)\right)<1$. Let $\psi$ be a measurable function of $[0, T]$ such that $0<\inf _{t \in[0, T]} \psi(t) \leq \sup _{t \in[0, T]} \psi(t)<\infty$. Then it is easy to see that $U(t, x) \triangleq \psi(t) \hat{U}(x)$ is a reasonably elastic utility random field. In particular, this example includes so-called discounted time-dependent utility functions of the form $U(t, x)=e^{-\beta t} \hat{U}(x)$.

EXAMPLE 3.3. Let $U_{1}:[0, T] \times \mathbb{R}_{+} \rightarrow \mathbb{R}$ be a deterministic utility field with corresponding $K_{1}$ and $K_{2}$ as in Definition 3.1(2). Further, let $U_{2}: \mathbb{R}_{+} \rightarrow \mathbb{R}$ be a utility function that satisfies

$$
U_{2}(\infty)>0, \quad \limsup _{x \rightarrow \infty}\left(\frac{x U_{2}^{\prime}(x)}{U_{2}(x)}\right)<1
$$

and

$$
0<\liminf _{x \rightarrow \infty}\left(\frac{U_{2}^{\prime}(x)}{K_{1}(x)}\right) \leq \limsup _{x \rightarrow \infty}\left(\frac{U_{2}^{\prime}(x)}{K_{1}(x)}\right)<\infty .
$$

One can check then the requirements of Definition 3.1 to see that

$$
U(t, x):= \begin{cases}U_{1}(t, x), & t<T, \\ U_{2}(x), & t=T,\end{cases}
$$

is a reasonably elastic utility random field.

EXAMPLE 3.4. Let $U_{1}:[0, T] \times \mathbb{R}_{+} \rightarrow \mathbb{R}$ be any deterministic reasonably elastic utility field and let $B_{t}$ be an adapted process uniformly bounded from above and away from zero. To model a stochastic discount factor, we define $U(t, x) \triangleq U_{1}\left(t, B_{t} x\right)$. Such a utility random field arises when the agent accrues utility from the nominal, instead of real, value of consumption.

With a utility random field $U$ we associate a random field $V: \Omega \times[0, T] \times$ $\mathbb{R}_{+} \rightarrow \mathbb{R}$ defined by

$$
V(t, y) \triangleq \sup _{x>0}[U(t, x)-x y], \quad 0<y<\infty,
$$

the conjugate of $U$. We also define the random field $I: \Omega \times[0, T] \times \mathbb{R}_{+} \rightarrow \mathbb{R}$ by $I(t, y)=\left(\partial_{2} U(t, \cdot)\right)^{-1}(y)$, the inverse marginal utility of $U$. The following proposition lists some important, though technical, properties of these random fields and their conjugates. They will be used extensively in the sequel. We leave their proofs to the care of the diligent reader.

PROPOSITION 3.5. Let $U$ be a utility random field and let $V$ be its conjugate.

1. There are (deterministic) utility functions $\underline{U}$ and $\bar{U}$ such that we have, a.s.,

$$
\underline{U}(x) \leq U(t, x) \leq \bar{U}(x) \quad \text { for all } x>0 \text { and all } t \in[0, T] .
$$


2. For a given $t \in[0, T]$, the function $V(t, \cdot)$ is finite-valued, strictly decreasing, strictly convex and continuously differentiable.

3. The convex conjugates $\underline{V}$ and $\bar{V}$ of $\underline{U}$ and $\bar{U}$ satisfy a.s.

$$
\underline{V}(y) \leq V(t, y) \leq \bar{V}(y) \quad \text { for all } y>0 \text { and all } t \in[0, T] .
$$

In particular, the function $t \mapsto V(t, y)$ is uniformly bounded for any $y \in(0, \infty)$.

DEFINITION 3.6. Any utility functions (i.e., strictly concave, strictly increasing and continuously differentiable functions that satisfy the Inada conditions) $\underline{U}: \mathbb{R}_{+} \rightarrow \mathbb{R}$ and $\bar{U}: \mathbb{R}_{+} \rightarrow \mathbb{R}$, such that $\underline{U}(x) \leq U(t, x) \leq \bar{U}(x)$ for all $x>0$ and $t \in[0, T]$, are called a minorant and a majorant of $U$, respectively. Functions $\underline{V}: \mathbb{R}_{+} \rightarrow \mathbb{R}$ and $\bar{V}: \mathbb{R}_{+} \rightarrow \mathbb{R}$ that are convex conjugates of some minorant and majorant of $U$ are called a minorant and a majorant of $V$, respectively.

REMARK 5. It follows immediately from the definition of convex conjugation that for any minorant and majorant $\underline{V}$ and $\bar{V}$ of $V$, we have $\underline{V}(y) \leq V(t, y) \leq \bar{V}(y)$ for all $y>0$ and $t \in[0, T]$.

Finally, we state a technical result that stems from the reasonable asymptotic elasticity condition; its proof is, mutatis mutandis, identical to the proof that leads to Corollary 6.3 on page 994 of [26].

PROPOSITION 3.7. Let $U$ be a utility random field. If we define the random sets

$$
\begin{aligned}
& \Gamma_{1}=\left\{\gamma>0: \exists x_{0}>0, \forall t \in[0, T], \forall \lambda>1, \forall x \geq x_{0}, U(t, \lambda x)<\lambda^{\gamma} U(t, x)\right\}, \\
& \Gamma_{2}=\left\{\gamma>0: \exists x_{0}>0, \forall t \in[0, T], \forall x \geq x_{0}, \partial_{2} U(t, x)<\gamma \frac{U(t, x)}{x}\right\}, \\
& \Gamma_{3}=\left\{\gamma>0: \exists y_{0}>0, \forall t \in[0, T], \forall 0<\rho<1, \forall 0<y \leq y_{0},\right. \\
& \left.V(t, \rho y)<\rho^{-\gamma /(1-\gamma)} V(t, y)\right\}, \\
& \Gamma_{4}=\left\{\gamma>0: \exists y_{0}>0, \forall t \in[0, T], \forall 0<y \leq y_{0},-\partial_{2} V(t, y)<\frac{\gamma}{1-\gamma} \frac{V(t, y)}{y}\right\},
\end{aligned}
$$

then $\inf \Gamma_{1}=\inf \Gamma_{2}=\inf \Gamma_{3}=\inf \Gamma_{4}=\mathrm{AE}[U]$ a.s.

3.2. The optimization problem and the main result. The principal task our agent is facing is how to control investment and consumption, in order to achieve maximal expected utility. At this point we have defined all notions necessary to cast this question in precise mathematical terms. 
PROBLEM 3.8. Let $U$ be a utility random field, let $\varepsilon$ be a cumulative endowment process and let $\mu$ be an admissible measure on $[0, T]$ as defined in Section 2.1. For any given initial capital $x>0$, we characterize the value function

$$
\mathfrak{U}(x) \triangleq \sup _{c \in \mathcal{A}^{\mu}(x+\mathcal{E})} \mathbb{E}\left[\int_{0}^{T} U(t, c(t)) \mu(d t)\right] \quad \text { (primal problem). }
$$

REMARK 6. When the above $(\mu \otimes \mathbb{P})$ integral fails to exist, we set its value to $-\infty$. This convention is equivalent to the approach taken in [21], where the authors considered only consumption processes such that the negative part $U^{-}(t, c(t))$ is $(\mu \otimes \mathbb{P})$-integrable. To avoid trivial situations, we adopt the following assumption throughout.

StAnding Assumption 3.9. There exists $x>0$ such that $\mathfrak{U}(x)<\infty$.

REMARK 7. Due to the boundedness of $\mathcal{E}_{T}$, the Assumption 3.9 holds under any conditions that guarantee the finiteness of the value function $\mathfrak{U}$ when $\mathcal{E}_{T} \equiv 0$. One such condition is given by $0 \leq U(t, x) \leq \kappa\left(1+x^{\alpha}\right) \forall x>0, t \in[0, T]$, for some constants $\kappa>0$ and $\alpha \in(0,1)$, in the model of [21]. For details, see Remark 3.9 on page 274 in [21] and compare with [23] and [38].

Together with the primal problem of (3.2), we set up the dual problem with value function

$$
\begin{gathered}
\mathfrak{V}(y) \triangleq \inf _{\mathbb{Q} \in \mathcal{D}} J(y, \mathbb{Q}) \text { where } \\
J(y, \mathbb{Q}) \triangleq \mathbb{E} \int_{0}^{T} V\left(t, y Y_{t}^{\mathbb{Q}}\right) \mu(d t)+y\left\langle\mathbb{Q}, \mathcal{E}_{T}\right\rangle, y>0 \quad \text { (dual problem). }
\end{gathered}
$$

It will be shown below that this dual problem is in fact well posed, that is, the integral in its definition always exists in $\overline{\mathbb{R}}$. Minimizing in (3.3) over the class $\mathscr{D}$, rather than over the smaller class $\mathcal{M}$, corresponds to the control-theoretic idea of "relaxing" the class of controls for the dual problem. This relaxation guarantees that the infimum in (3.3) is attained, and this in turn leads to a consumption-rate process that attains the supremum in the primal problem of (3.2). This is the gist of the main result of this article, which can be stated as follows:

THEOREM 3.10. Let $\mathcal{E}=\left(\mathcal{E}_{t}\right)_{t \in[0, T]}$ be a cumulative endowment process and let $\mu$ be an admissible measure. Furthermore, let $U$ be a utility random field, let $V$ be its conjugate, and let $\mathfrak{U}$ and $\mathfrak{V}$ be the value functions of the primal and the dual problems, respectively. Under Assumption 3.9, the following assertions hold:

(i) $|\mathfrak{U}(x)|<\infty$ for all $x>0$ and $|\mathfrak{V}(y)|<\infty$ for all $y>0$, that is, the value functions are finite throughout their domains. 
(ii) The value functions $\mathfrak{U}$ and $\mathfrak{V}$ are continuously differentiable, $\mathfrak{U}$ is strictly concave and $\mathfrak{V}$ is strictly convex.

(iii) $\mathfrak{U}(x)=\inf _{y>0}[\mathfrak{V}(y)+x y]$ and $\mathfrak{V}(y)=\sup _{x>0}[\mathfrak{U}(x)-x y]$ for $x, y>0$, that is, $\mathfrak{U}$ and $\mathfrak{V}$ are convex conjugates of each other.

(iv) The derivatives $\mathfrak{U}^{\prime}$ and $\mathfrak{V}^{\prime}$ of the value functions satisfy $\lim _{y \downarrow 0} \mathfrak{V}^{\prime}(y)=$ $-\lim _{x \downarrow 0} \mathfrak{U}^{\prime}(x)=0=-\infty$,

$$
\lim _{y \rightarrow 0}\left(-\mathfrak{V}^{\prime}(y)\right)=-\lim _{x \rightarrow \infty} \mathfrak{U}^{\prime}(x) \in\left[\inf _{\mathbb{Q} \in \mathscr{D}}\left\langle\mathbb{Q}, \mathcal{E}_{T}\right\rangle, \sup _{\mathbb{Q} \in \mathscr{D}}\left\langle\mathbb{Q}, \mathcal{E}_{T}\right\rangle\right] .
$$

(v) Both primal and dual problems have solutions $\hat{c}^{x}(\cdot) \in \mathcal{A}^{\mu}(x+\mathcal{E})$ and $\hat{\mathbb{Q}}^{y} \in \mathcal{D}$, respectively, for all $x, y>0$. For $x>0$ and $y>0$ related by $\mathfrak{U}^{\prime}(x)=y$, we have

$$
\hat{c}^{x}(t)=I\left(t, y Y_{t}^{\hat{\mathbb{Q}}^{y}}\right), \quad 0 \leq t \leq T,
$$

where $\hat{\mathbb{Q}}^{y}$ is a solution to the dual problem corresponding to y. Furthermore, $\hat{c}^{x}(\cdot)$ is the unique optimal consumption-rate process and $\hat{\mathbb{Q}}^{y}$ is determined uniquely as far as the process $Y^{\hat{\mathbb{Q}}^{y}}$ and the action of $\hat{\mathbb{Q}}^{y}$ on $\mathcal{E}_{T}$ are concerned.

(vi) With $\hat{\mathbb{Q}}^{y} \in \mathcal{D}$ as in (v), the derivative $\mathfrak{V}^{\prime}(y)$ satisfies

$$
\mathfrak{V}^{\prime}(y)=\left\langle\hat{\mathbb{Q}}^{y}, \mathcal{E}_{T}\right\rangle-\mathbb{E}\left[\int_{0}^{T} Y_{t}^{\hat{\mathbb{Q}}^{y}} I\left(t, y Y_{t}^{\hat{\mathbb{Q}}^{y}}\right) \mu(d t)\right] .
$$

EXAMPLE 3.11. Let $U_{1}$ be a utility random field and let $U_{2}$ be a utility function. Consider the problem of maximizing expected utility from consumption and terminal wealth,

$$
\mathfrak{U}(x):=\sup \left(\mathbb{E}\left[\int_{0}^{T} U_{1}(t, c(t)) d t+U_{2}\left(X_{T}\right)\right]\right),
$$

where the supremum is taken over all admissible investment-consumption strategies. This problem can be regarded as a special case of our primal problem. Indeed, if we view the terminal wealth as being consumed instantaneously, we can translate (3.4) into

$$
\mathfrak{U}(x)=\sup _{c \in \mathcal{A}^{\mu}(x+\mathcal{E})} \mathbb{E}\left[\int_{0}^{T} U(t, c(t)) \mu(d t)\right],
$$

where $\mu=\frac{1}{2 T} \lambda+\frac{1}{2} \delta_{\{T\}}, \lambda$ denotes the Lebesgue measure on $[0, T]$ and

$$
U(t, x):= \begin{cases}2 T U_{1}\left(t, \frac{x}{2 T}\right), & t<T, \\ 2 U_{2}\left(\frac{x}{2}\right), & t=T,\end{cases}
$$

provided $U_{1}$ and $U_{2}$ satisfy the requirements of Example 3.3. In this context, $C_{T}-C_{T-}=\frac{1}{2} c(T)$ plays the role of terminal wealth. 


\section{Examples.}

4.1. The Itô process model. We specialize the specifications of our model as follows: Let $\left(\Omega, \mathcal{F},\left(\mathcal{F}_{t}\right)_{t \in[0, T]}, \mathbb{P}\right)$ be a filtered probability space that supports a $d$-dimensional Brownian motion $W=\left(W_{t}\right)_{t \in[0, T]}$ and assume that $\mathbb{F} \triangleq\left(\mathcal{F}_{t}\right)_{t \in[0, T]}$ is the augmentation of the filtration generated by $W$. The market coefficients are given by a bounded real-valued interest rate process $r$, a bounded appreciation rate process $b$ that takes values in $\mathbb{R}^{d}$ and a $(d \times d)$ matrix-valued volatility process $\sigma$. We assume that $r, b$ and $\sigma$ are progressively measurable and $\sigma(t)$ is a symmetric nonsingular matrix for each $t$, with all eigenvalues uniformly bounded from above and away from zero, almost surely. [When $r(t), b(t)$ and $\sigma(t)$ are nonanticipative functionals of past and present stock prices $(S(u))_{u \in[0, t]}$ for each $t \in[0, T]$ and the resulting stochastic differential equation (4.1) for $S$ has a strong solution, the nondegeneracy of $\sigma(t)$ implies that $S$ and the driving Brownian motion $W$ generate the same filtration. In this case, consumption-investment decisions are based solely on observations of (past and present) stock prices.] The dynamics of the money market (numeraire) $B$ and the stock prices $S$ are

$$
\begin{aligned}
d B_{t} & =B_{t} r(t) d t, \quad B_{0}=1, \\
d S_{t} & =\operatorname{diag}\left(S_{t}\right)\left[b(t) d t+\sigma(t) d W_{t}\right], \quad S_{0}=s_{0} \in \mathbb{R}_{++}^{d} .
\end{aligned}
$$

With the $d$-dimensional vector $\mathbf{1}_{d} \triangleq(1,1, \ldots, 1)^{\prime}$, we define the market price of risk:

$$
\theta(t)=\sigma^{-1}(t)\left[b(t)-r(t) \mathbf{1}_{d}\right] .
$$

The equations in (4.1) specify a complete market model which, however, becomes incomplete when the cone $\mathcal{K}$ of portfolio constraints is introduced. Then the set $\mathcal{y}^{\mathcal{M}}$ of (2.10) satisfies

$$
y^{\mathcal{M}} \subseteq\left\{Z_{v}(\cdot): v \in \mathbb{K} \text { and } Z_{v}(\cdot) \text { is a positive martingale }\right\}
$$

(see [23], page 712; [7], page 777; [15], page 50). Here $\mathbb{K}$ is the set of all progressively measurable processes $v:[0, T] \times \Omega \rightarrow \mathbb{R}^{d}$ such that

$$
\int_{0}^{T}\|v(t)\|^{2} d t<\infty \quad \text { and } \quad v(t)^{\prime} p \geq 0 \quad \forall p \in \mathcal{K}, t \in[0, T],
$$

hold almost surely (i.e., $v$ takes values in the barrier cone of $-\mathcal{K}$ ) and

$$
Z_{v}(\cdot) \triangleq \exp \left(-\int_{0}^{\cdot}\left(\theta(t)+\sigma^{-1}(t) v(t)\right)^{\prime} d W_{t}-\frac{1}{2} \int_{0}^{\cdot}\left\|\theta(t)+\sigma^{-1}(t) v(t)\right\|^{2} d t\right) .
$$

Let us recall also the sets $y^{\mathscr{D}}$ and $\mathcal{y}^{\mathcal{M}}$ of (2.6). In the following proposition we characterize the subset $\mathcal{Y}_{\max }^{\mathcal{D}}$ of the dual domain $y^{\mathcal{D}}$, which consists of processes that are strictly positive on the support supp $\mu$ and maximal; that is, not dominated by any other process in $\mathcal{Y}^{\mathcal{D}}$. We recall that $\operatorname{supp} \mu$ is defined to be $[0, T]$ if $\mu$ charges $\{T\}$, and to be $[0, T)$ otherwise. 
PROPOSITION 4.1. The elements of $\mathcal{Y}_{\max }^{\mathcal{D}}$ are local martingales of the form

$$
\mathbb{P}\left[Y_{t}=Z_{v}(t), \forall t \in \operatorname{supp} \mu\right]=1 \quad \text { for some } v \in \mathbb{K} .
$$

PROOF. For simplicity of notation, and without loss of generality, we assume in this proof that $r(\cdot)$ and $b(\cdot)$ are identically equal to zero in (4.1), that the volatility $\sigma(\cdot)$ is the identity matrix and that $\operatorname{supp} \mu=[0, T]$.

Let $Y \in \mathcal{Y}_{\max }^{\mathcal{D}}$ be a maximal element of the "dual domain" $y^{\mathcal{D}}$. From the multiplicative decomposition theorem for positive special semimartingales (see [19], Propositions 6.19 and 6.20), there exist a continuous local martingale $M$ with $M_{0}=1$ and a nonincreasing, predictable process $D$ with RCLL paths, $D_{0}=1$ and $D_{T}>0$ a.s., such that $Y_{t}=M_{t} \cdot D_{t}, 0 \leq t \leq T$. By the martingale representation theorem for the Brownian filtration (see [20], Theorem 3.4.15 and Problem 3.4.16), there is an $\mathbb{F}$-progressively measurable process $v:[0, T] \times$ $\Omega \rightarrow \mathbb{R}^{d}$ with $\int_{0}^{T}\|v(s)\|^{2} d s<\infty$ a.s. such that

$$
M_{t}=Z_{v}(t) \equiv \exp \left(-\int_{0}^{t} v(s)^{\prime} d W(s)-\frac{1}{2} \int_{0}^{t}\|v(s)\|^{2} d s\right), \quad 0 \leq t \leq T .
$$

For any admissible trading strategy $H$ and $x>0$ such that

$$
X_{t}^{x, H} \triangleq x+\int_{0}^{t} H_{u}^{\prime} \operatorname{diag}\left(S_{u}\right) d W_{u} \geq 0 \quad \forall t \in[0, T]
$$

holds almost surely, the process $Y X^{x, H}$ is a supermartingale by Theorem 2.10.

By Itô's formula (e.g., [31], Section II.7) we have

$$
d\left(Y_{t} X_{t}^{x, H}\right)=X_{t}^{x, H} d Y_{t}+Y_{t-} d X_{t}^{x, H}+d\left[X^{x, H}, Y\right]_{t}
$$

and $d Y_{t}=M_{t} d D_{t}+D_{t-} d M_{t}+d[M, D]_{t}$. Since $M$ is continuous, and $D$ is predictable and of finite variation, $d[M, D]_{t} \equiv 0$, so $d Y_{t}=M_{t} d D_{t}-D_{t-} M_{t} v(t) d W_{t}$, because $d M_{t}=-M_{t} v(t)^{\prime} d W_{t}$. Furthermore, $d\left[X^{x, H}, Y\right]_{t}=-D_{t-} M_{t} H_{t}^{\prime} \times$ $\operatorname{diag}\left(S_{t}\right) v(t) d t$ from (4.2). It follows that

$$
d\left(Y_{t} X_{t}^{x, H}\right)=d L_{t}+M_{t}\left[X_{t}^{x, H} d D_{t}-D_{t-} H_{t}^{\prime} \operatorname{diag}\left(S_{t}\right) v(t) d t\right]
$$

where $L$ is a local martingale.

Now we prove that $v \in \mathbb{K}$. To do that, let us assume to the contrary that $v$ fails to satisfy the relationship

$$
v(t)^{\prime} p \geq 0 \quad \text { for all } p \in \mathcal{K}, \lambda \otimes \mathbb{P} \text {-a.s. }
$$

Then we can find a constant $\varepsilon>0$, a predictable set $A$ such that $(\lambda \otimes \mathbb{P})(A)>0$ and a bounded predictable process $\hat{H}$ taking values in $\mathcal{K}$, such that $\hat{H}=0$ off $A$ and

$$
D_{t-v}(t)^{\prime} \operatorname{diag}\left(S_{t}\right) \hat{H}_{t} \leq-\varepsilon \quad \text { on } A \text {. }
$$


We can also assume that $\left\|\operatorname{diag}\left(S_{t}\right) \hat{H}_{t}\right\|=1$ on $A,(\lambda \otimes \mathbb{P})$-a.s. For any $x>0$, we introduce the first hitting time $T^{x} \triangleq \inf \left\{t \in[0, T): X_{t}^{x, \hat{H}}=0\right\} \wedge T$, and the process $H_{t}^{x} \triangleq \hat{H}_{t} \mathbf{1}_{\left[0, T^{x}\right]}(t)$, so that $X_{t}^{x, H^{x}} \geq 0$ for all $t \in[0, T]$ a.s. Now we have all the ingredients to define the family of signed measures $\left\{\varphi_{x}\right\}_{x>0}$, given by

$$
\varphi_{x}(B) \triangleq \mathbb{E}\left(\int_{0}^{T} \mathbf{1}_{B}(t) X_{t}^{x, H^{x}} d D_{t}+\varepsilon \int_{0}^{T} \mathbf{1}_{B}(t) d t\right),
$$

on the $\sigma$-algebra of $\mathbb{F}$-predictable subsets of $[0, T] \times \Omega$. By the supermartingale property of $Y X^{x, H^{x}}$, the relationship (4.3) and (4.5), and the strict positivity of the process $M$, we have $\varphi_{x}(B) \leq 0$ for any $x>0$ and any $\mathbb{F}$-predictable set $B \subseteq A \cap\left[0, T^{x}\right]$. Due to the fact that $H^{x}$ is zero off $A$, the set $A \cap\left[0, T^{x}\right]$ is still of positive $(\mu \otimes \mathbb{P})$ measure. By Theorem 2.1 of [10], there exists an $\mathbb{F}$-predictable process $g:[0, T] \times \Omega \rightarrow \mathbb{R}$ and an $\mathbb{F}$-predictable set $N \subseteq[0, T] \times \Omega$ such that

$$
\begin{aligned}
& D_{t}=\int_{0}^{t} g(u) d u+\int_{0}^{t} \mathbf{1}_{N}(s) d D_{u} \quad \text { and } \\
& \int_{0}^{t} \mathbf{1}_{N}(u) d u=0 \quad \text { for all } t \in[0, T]
\end{aligned}
$$

and $\int_{0}^{T} g(u) d u \leq D_{T} \leq 1$ hold almost surely. From the definition (4.6) of $\varphi_{x}$ and the decomposition (4.7), for any $x>0$ and any predictable $B \subseteq\left(A \cap\left[0, T^{x}\right]\right) \backslash N$, we have

$$
0 \geq \varphi_{x}(B)=\mathbb{E} \int_{0}^{T}\left(X_{t}^{x, H^{x}} g(t)+\varepsilon\right) \mathbf{1}_{B}(t) d t .
$$

Equation (4.7) asserts $(\lambda \otimes \mathbb{P})(N)=0$ for all $x>0$, so the above inequality implies that $X_{t}^{x, H^{x}} g(t)+\varepsilon \leq 0$ holds $(\lambda \otimes \mathbb{P})$-a.e. on $A \cap\left[0, T^{x}\right]$, for any $x>0$. We observe that the right-continuous inverse $Q^{-1}$ of the process

$$
\begin{aligned}
Q_{t} & \triangleq \int_{0}^{t} \mathbf{1}_{A}(u) d u \\
& =\int_{0}^{t}\left\|\operatorname{diag}\left(S_{u}\right) \hat{H}_{u}\right\|^{2} d u=\left[X^{0, \hat{H}}, X^{0, \hat{H}}\right]_{t}, \quad 0 \leq t \leq T,
\end{aligned}
$$

is a random time change, which transforms the process $X^{0, \hat{H}}$ into a $\mathbb{G}$-Brownian motion $\xi_{s} \triangleq X_{Q_{s}^{-1}}^{0, \hat{H}}$ on the stochastic interval $\Theta \triangleq\left[0, Q_{T}\right.$ ), with $\mathcal{g}_{s} \triangleq \mathcal{F}_{Q_{s}^{-1}}$ (see Theorem 4.6 on page 174 and Problem 4.7 on page 175 in [20]). Let $f(s)$ be the composite process $g\left(Q_{s}^{-1}\right)$ and let $R_{x}=Q_{T^{x}}$ be the first hitting time of $-x$ by the $\mathbb{G}$-Brownian motion $\xi$. Thus, for any $x>0$ and any $\mathbb{G}$-predictable set $B \subseteq \Theta \cap\left[0, R^{x}\right]$, we have

$$
\begin{aligned}
1 & \geq-\int_{0}^{T} \mathbf{1}_{A \cap\left[0, T^{x}\right]}(t) g(t) d t \\
& \geq-\int_{0}^{Q_{T}} \mathbf{1}_{B}(s) f(s) d s \geq \varepsilon \int_{0}^{Q_{T}} \mathbf{1}_{B}(s) \frac{1}{x+\xi_{s}} d s \quad \text { a.s. }
\end{aligned}
$$


Relationship (4.9) implies that $x+\xi_{s} \geq \varepsilon,(\lambda \otimes \mathbb{P})$-a.e. on $\Theta \cap\left[0, R^{x}\right]$. This contradicts the fact that $\mathbb{P}\left(x+\xi_{R_{x}}=0\right)>0$ and, for small enough $x, \mathbb{P}\left(R_{x} \in\right.$ $\Theta)>0$.

Therefore, relationship (4.4) holds and we know that the process $M$ dominates $Y$. By truncation, $M$ can be obtained as the Fatou limit of a sequence of martingales in $\mathcal{Y}^{\mathcal{M}}$, so by Theorem $2.9, M \in \mathcal{Y}^{*}$. Theorem 2.10 states that $M$ is dominated by an element of $y^{\mathcal{D}}$. Since $M$ is a local martingale with $M_{0}=1$ and all elements $Y \in \mathcal{Y}^{\mathcal{D}}$ are supermartingales with $Y_{0} \leq 1$, we can find a sequence $\left\{T_{n}\right\}_{n \in \mathbb{N}}$ of stopping times that reduces $M$ and use it to conclude that $M \in \mathcal{Y}_{\max }^{\mathcal{D}} \subseteq \mathcal{Y}^{\mathscr{D}}$ and $Y=M$.

Because the optimal solution of the dual problem must be positive on supp $\mu$, we have the following corollary:

COROLlARY 4.2. In the setting of an Itô process model, the primal problem has

$$
c(t)=I\left(t, y Z_{v}(t) D_{t}\right), \quad 0 \leq t \leq T
$$

as the optimal consumption-rate process for some constant $y>0$, some predictable process $v$ with values in the barrier cone of $-\mathcal{K}$ and $\int_{0}^{T}\|v(s)\|^{2} d s<\infty$ a.s., and some positive, nonincreasing and $\mathcal{F}$-predictable process $D$ with $D_{0} \leq 1$. Both processes $Z_{v}$ and $Z_{v} D$ are in $y^{D}$.

REMARK 8. Note that Corollary 4.2 answers affirmatively a question posed in Remark 5.8 on page 290 of [21], where it was stressed that the problem of maximizing expected utility from consumption only "is not well understood" in an incomplete/constrained market, even with deterministic endowment.

COROLlary 4.3. Suppose that the terminal value of the endowment process is "attainable": $\varepsilon_{T}=X_{T}=x+\int_{0}^{T} H_{u}^{\prime} d S_{u}$ for some $X \in \mathcal{X}$ as in (2.2). This is the case, for instance, if the market model is complete or if $\mathcal{E}_{T}$ is nonrandom. Then the optimal consumption process of (4.10) takes the simple form

$$
c(t)=I\left(t, y Z_{v}(t)\right), \quad 0 \leq t \leq T .
$$

This is because we have then $\left\langle\mathbb{Q}, \mathcal{E}_{T}\right\rangle=x$ for every $\mathbb{Q} \in \mathscr{D}$ (cf. Remark 4.4 in [8]) and thus the dual objective function $\mathbb{Q} \mapsto J(y, \mathbb{Q})=\mathbb{E} \int_{0}^{T} V\left(t, y Y_{t}^{\mathbb{Q}}\right) \times$ $\mu(d t)+x y$ of (3.3) becomes monotone in $Y^{\mathbb{Q}}$. As a result, the optimal consumption in (4.10) has $D \equiv 1$. It would be very interesting to find more general conditions that guarantee an optimal consumption process $c(\cdot)$ of this simple form. 
4.2. Optimal consumption of a random endowment. In this example we consider a situation in which the agent must optimally consume an uncertain future endowment stream without any possibility of hedging the uncertainty in a financial market. This problem was studied by [27] in the special case of a point-process setting. We consider the following general version:

Problem 4.4. Let $\left(\Omega, \mathcal{F},(\mathcal{F})_{t \in[0, T]}, \mathbb{P}\right)$ be a filtered probability space that satisfies the usual hypotheses and let $\varepsilon(\cdot)$ be a nonnegative progressively measurable process such that $\varepsilon_{T}=\int_{0}^{T} \varepsilon(t) d t$ is uniformly bounded away from both zero and infinity. With a given utility function $U$, the goal is to find a progressively measurable, nonnegative consumption-rate process $c(\cdot)$ so as to maximize the expected utility $\mathbb{E} \int_{0}^{T} U(c(t)) d t$, subject to the stringent budget constraint

$$
\int_{0}^{T} c(t) d t \leq \int_{0}^{T} \varepsilon(t) d t \quad \text { a.s. }
$$

The following theorem was proved in [27]. We include a proof for the reader's convenience and denote by $I(\cdot)$ the inverse marginal utility: $I(y)=\left(U^{\prime}\right)^{-1}(y)$ for $0<y<\infty$.

THEOREM 4.5. Suppose there exists a positive $\mathbb{F}$-martingale $Y$ such that

$$
\int_{0}^{T} I\left(Y_{t}\right) d t=\int_{0}^{T} \varepsilon(t) d t
$$

holds almost surely. Then an optimal consumption process is given by

$$
\hat{c}(t)=I\left(Y_{t}\right), \quad 0 \leq t \leq T .
$$

PROOF. From the inequality $U(I(y)) \geq U(c)+y I(y)-y c$, valid for $y>0$ and $c>0$, we obtain

$$
U\left(I\left(Y_{t}\right)\right) \geq U(c(t))+Y_{t} I\left(Y_{t}\right)-Y_{t} c(t)
$$

for every positive, adapted process $c(\cdot)$. Therefore,

$$
\mathbb{E} \int_{0}^{T} U(\hat{c}(t)) d t \geq \mathbb{E} \int_{0}^{T} U(c(t)) d t+\mathbb{E} \int_{0}^{T} Y_{t} I\left(Y_{t}\right) d t-\mathbb{E} \int_{0}^{T} Y_{t} c(t) d t
$$

and the optimality of the process $\hat{c}(\cdot)$ in (4.13), among those that satisfy (4.11), follows once we have shown that this latter constraint implies

$$
\mathbb{E} \int_{0}^{T} Y_{t} I\left(Y_{t}\right) d t \geq \mathbb{E} \int_{0}^{T} Y_{t} c(t) d t
$$


To do that, it suffices to introduce the probability measure $\tilde{\mathbb{P}}(A) \triangleq \frac{1}{y} \mathbb{E}\left[Y_{T} \mathbf{1}_{A}\right]$ for $A \in \mathcal{F}_{T}$, where $y=\mathbb{E}\left[Y_{0}\right] \in(0, \infty)$. This measure is equivalent to $\mathbb{P}$; thus, the martingale property of $Y,(4.12)$ and (4.11) lead to (4.14), because

$$
\begin{aligned}
\mathbb{E} \int_{0}^{T} Y_{t} I\left(Y_{t}\right) d t & =y \cdot \tilde{\mathbb{E}} \int_{0}^{T} I\left(Y_{t}\right) d t=y \cdot \tilde{\mathbb{E}} \int_{0}^{T} \varepsilon(t) d t \\
& \geq y \cdot \tilde{\mathbb{E}} \int_{0}^{T} c(t) d t=\mathbb{E} \int_{0}^{T} Y_{t} c(t) d t .
\end{aligned}
$$

We prove the following existence result, which is a partial converse of Theorem 4.5.

PROPOSITION 4.6. When the utility function $U(\cdot)$ satisfies the "reasonable asymptotic elasticity" condition of Definition 3.1(4), the optimization Problem 4.4 has a unique solution which is of the form $\hat{c}(t)=I\left(Y_{t}\right), 0 \leq t \leq T$, for some positive RCLL supermartingale $Y$. This process satisfies

$$
\int_{0}^{T} I\left(Y_{t}\right) d t=\int_{0}^{T} \varepsilon(t) d t \quad \text { a.s. }
$$

PROOF. We note first that Problem 4.4 is a special case of our primal problem with a one-dimensional stock price process $S_{t} \equiv 1$ and trivial bond price process $B_{t} \equiv 1$. In this case all measures equivalent to $\mathbb{P}$ are equivalent supermartingale measures and, by Theorem 2.10, any RCLL supermartingale $Y$ with $Y_{0} \leq 1$ is in $y^{*}$. By Theorem 3.10, the unique optimal consumption-rate process is given by $\hat{c}(t)=I\left(y Y_{t}^{\hat{\mathbb{Q}}}\right), 0 \leq t \leq T$, for some $y>0$ and some $\hat{\mathbb{Q}} \in \mathcal{D}$. To finish the proof, we define $Y_{t}=y Y_{t}^{\hat{\mathbb{Q}}}$ and note that Proposition 2.13 implies

$$
\int_{0}^{T} \hat{c}(t) d t \leq \int_{0}^{T} \varepsilon(t) d t \quad \text { a.s. }
$$

because every measure equivalent to $\mathbb{P}$ is in $\mathcal{M}$. From Theorem 3.10 (v) and (vi), it follows that, for the optimal solution $\hat{\mathbb{Q}} \in \mathcal{D}$ of the dual problem, we have

$$
\begin{aligned}
\mathbb{E} \int_{0}^{T} Y_{T}^{\hat{\mathbb{Q}}} \hat{c}(t) d t & =\left\langle\hat{\mathbb{Q}}, \int_{0}^{T} \hat{c}(t) d t\right\rangle \\
& \geq\left\langle\hat{\mathbb{Q}}, \int_{0}^{T} \varepsilon(t) d t\right\rangle=\mathbb{E} \int_{0}^{T} Y_{T}^{\hat{\mathbb{Q}}} \varepsilon(t) d t .
\end{aligned}
$$

The random variable $Y_{T}^{\hat{\mathbb{Q}}}=L_{T}^{\hat{\mathbb{Q}}}=\frac{d(\hat{\mathbb{Q}})^{r}}{d \mathbb{P}}$ is strictly positive, so (4.15) follows from (4.16) and (4.17). 


\section{APPENDIX}

Proof of the main result, Theorem 3.10. In this part we state and prove a number of results that lead to the proof of our main result, Theorem 3.10. To simplify the notation, we do not relabel the indices when passing to a subsequence.

A.1. Existence in the dual problem. We study the dual problem first. In this subsection we point out some properties of the dual objective function and establish the existence of $\hat{\mathbb{Q}} \in \mathcal{D}$, which is optimal in the dual problem of (3.3). The negative part $\max \{0,-V\}$ of the random field $V$, the convex conjugate of $U$ introduced in (3.1), is denoted by $V^{-}$. Our first result establishes a lower semicontinuity property for the nonlinear part of the dual objective function.

LEMMA A.1. For $y>0$, the family of random processes $\left\{V^{-}\left(\cdot, y Y^{\mathbb{Q}}\right)\right.$ : $\mathbb{Q} \in \mathscr{D}\}$ is uniformly integrable with respect to the product measure $(\mu \otimes \mathbb{P})$ on $[0, T] \times \Omega$. Furthermore, we have the lower semicontinuity relationship

$$
\mathbb{E}\left[\int_{0}^{T} V\left(t, y Y_{t}^{\mathbb{Q}}\right) \mu(d t)\right] \leq \liminf _{n} \mathbb{E}\left[\int_{0}^{T} V\left(t, y Y_{t}^{\mathbb{Q}^{(n)}}\right) \mu(d t)\right]
$$

for all $\left\{\mathbb{Q}^{(n)}\right\}_{n \in \mathbb{N}} \subseteq \mathbb{D}$ such that $\left\{Y^{\mathbb{Q}^{(n)}}\right\}$ converges $(\mu \otimes \mathbb{P})$-a.e. to a $R C L L$ supermartingale $Y^{\mathbb{Q}}$.

PRoof. Let $\underline{V}(\cdot)$ be a minorant of $V(\cdot, \cdot)$ as introduced in Definition 3.6. We define $\varphi: \mathbb{R}_{+} \rightarrow \mathbb{R}_{+}$to be the right-continuous inverse of $\underline{V}^{-}(\cdot)$, that is, $\varphi(x) \triangleq \inf \left\{y \geq 0: \underline{V}^{-}(y)<x\right\}$ for $x \geq 0$. Suppose first that $\varphi(x)$ is finite for all $x \geq 0$. Then, by l'Hôpital's rule,

$$
\lim _{x \rightarrow \infty} \frac{\varphi(x)}{x}=\lim _{x \rightarrow \infty} \varphi^{\prime}(x)=\lim _{y \rightarrow \infty} \frac{1}{\left(\underline{V}^{-}\right)^{\prime}(y)}=\infty .
$$

The family $\left\{\varphi\left(V^{-}\left(\cdot, y Y_{.}^{\mathbb{Q}}\right)\right): \mathbb{Q} \in \mathbb{D}\right\}$ is bounded in $L^{1}(\mu \otimes \mathbb{P})$, because

$$
\begin{aligned}
\mathbb{E}\left[\int_{0}^{T} \varphi\left(V^{-}\left(t, y Y_{t}^{\mathbb{Q}}\right)\right) \mu(d t)\right] & \leq \mathbb{E}\left[\int_{0}^{T} \varphi\left(\underline{V}^{-}\left(y Y_{t}^{\mathbb{Q}}\right)\right) \mu(d t)\right] \\
& \leq \varphi(0)+\mathbb{E}\left[\int_{0}^{T} y Y_{t}^{\mathbb{Q}} \mu(d t)\right] \leq \varphi(0)+y .
\end{aligned}
$$

Thus, by the theorem of de la Vallée Poussin (see [35], Lemma II.6.3, page 190), the family of processes $\left\{\varphi\left(V^{-}\left(\cdot, y Y_{.}^{\mathbb{Q}}\right)\right): \mathbb{Q} \in \mathscr{D}\right\}$ is $(\mu \otimes \mathbb{P})$-uniformly integrable. If $\varphi(x)=\infty$ for some $x>0$, then $\underline{V}^{-}(\cdot)$ is a bounded function and uniform integrability follows readily. 
Let $\left\{\mathbb{Q}^{(n)}\right\}_{n \in \mathbb{N}} \subseteq \mathcal{D}$ be a sequence such that $\left\{Y^{\mathbb{Q}^{(n)}}\right\}_{n \in \mathbb{N}}$ converges to a RCLL supermartingale $Y^{\mathbb{Q}},(\mu \otimes \mathbb{P})$-a.e. By uniform integrability we have that

$$
\begin{aligned}
& \mathbb{E}\left[\int_{0}^{T} V^{-}\left(t, y Y_{t}^{\mathbb{Q}^{(n)}}\right) \mu(d t)\right] \\
& \quad \rightarrow \mathbb{E}\left[\int_{0}^{T} V^{-}\left(t, y Y_{t}^{\mathbb{Q}}\right) \mu(d t)\right] \quad \text { as } n \rightarrow \infty .
\end{aligned}
$$

As for the positive parts, Fatou's lemma gives

$$
\liminf _{n} \mathbb{E}\left[\int_{0}^{T} V^{+}\left(t, y Y_{t}^{\mathbb{Q}^{(n)}}\right) \mu(d t)\right] \geq \mathbb{E}\left[\int_{0}^{T} V^{+}\left(t, y Y_{t}^{\mathbb{Q}}\right) \mu(d t)\right] .
$$

The claim now follows from (A.2) and (A.3).

The following result establishes the existence of a solution to the dual problem.

Proposition A.2. For each $y>0$ such that $\mathfrak{V}(y)<\infty$, there is $\hat{\mathbb{Q}} \in \mathcal{D}$ such that

$$
\mathfrak{V}(y)=J(y, \hat{\mathbb{Q}})=\mathbb{E}\left[\int_{0}^{T} V\left(t, y Y_{t}^{\hat{\mathbb{Q}}}\right) \mu(d t)\right]+y\left\langle\hat{\mathbb{Q}}, \mathcal{E}_{T}\right\rangle .
$$

Proof. We fix $y>0$ and let $\left\{\mathbb{Q}^{(n)}\right\}_{n \in \mathbb{N}}$ be a minimizing sequence for $J(y, \cdot)$. We first assume that the sequence $\left\{\left\langle\mathbb{Q}^{(n)}, \mathcal{E}_{T}\right\rangle\right\}_{n \in \mathbb{N}}$ converges in $\mathbb{R}$. This can be justified by extracting a subsequence, if necessary. By Lemma 5.2 in [16] we can find a sequence of convex combinations of elements in $\left\{Y^{\mathbb{Q}^{(n)}}\right\}_{n \in \mathbb{N}}$, which converges to a RCLL supermartingale $Y$ in the Fatou sense. Because of boundedness in $\mathbb{L}^{1}(\mu \otimes \mathbb{P})$, we can pass (thanks to Komlós's theorem) to a subsequence of convex combinations to achieve convergence $(\mu \otimes \mathbb{P})$-a.e. By Proposition 2.6, the limit is still $Y$. Because of the convexity of $V(t, \cdot)$ and the convergence of the sequence $\left\{\left\langle\mathbb{Q}^{(n)}, \mathcal{E}_{T}\right\rangle\right\}_{n \in \mathbb{N}}$, passing to convex combinations preserves the property of being a minimizing sequence. By Proposition 2.6, the limit $Y$ is of the form $Y \hat{\mathbb{Q}}$ for some (and then every) cluster point $\hat{\mathbb{Q}}$ of $\left\{\mathbb{Q}^{(n)}\right\}_{n \in \mathbb{N}}$; the existence of such a cluster point is guaranteed by Alaoglu's theorem ([37], Theorem 2.A.9). Invoking Lemma A.1 establishes the claim of the proposition.

A.2. Conjugacy and finiteness of $\mathfrak{U}(\cdot)$ and $\mathfrak{V}(\cdot)$. The next step is to establish a conjugacy relationship between $\mathfrak{U}(\cdot)$ and $\mathfrak{V}(\cdot)$. The most important tool in this endeavor is the minimax theorem.

LEMMA A.3. The function $\mathfrak{V}(\cdot)$ is the convex conjugate of $\mathfrak{U}(\cdot)$, namely: $\mathfrak{V}(y)=\sup _{x>0}[\mathfrak{U}(x)-x y]$ for $y>0$. 
Proof. For fixed $y \in(0, \infty)$ and $n \in \mathbb{N}$, let $s_{n}$ denote the set of all nonnegative, progressively measurable processes $c:[0, T] \times \Omega \rightarrow[0, n]$. The sets $\delta_{n}$ can be viewed as closed subsets of balls in $\mathbb{L}^{\infty}(\mu \otimes \mathbb{P})$. Thanks to the concavity of $U(t, \cdot)$, the compactness of $s_{n}$ (by Alaoglu's theorem) and the convexity of $\mathscr{D}$, we can use the minimax theorem (see [36], Theorem 45.8 and its corollaries) to obtain

$$
\begin{aligned}
\sup _{c \in \S_{n}} & \inf _{\mathbb{Q} \in \mathcal{D}}\left(\mathbb{E} \int_{0}^{T}\left(U(t, c(t))-y Y_{t}^{\mathbb{Q}} c(t)\right) \mu(d t)+y\left\langle\mathbb{Q}, \mathcal{E}_{T}\right\rangle\right) \\
& =\inf _{\mathbb{Q} \in D} \sup _{c \in \delta_{n}}\left(\mathbb{E} \int_{0}^{T}\left(U(t, c(t))-y Y_{t}^{\mathbb{Q}} c(t)\right) \mu(d t)+y\left\langle\mathbb{Q}, \mathcal{E}_{T}\right\rangle\right)
\end{aligned}
$$

for any $n \in \mathbb{N}, y>0$. Proposition 2.13 guarantees that $\bigcup_{x>0} \mathscr{A}^{\mu}(x+\mathcal{E})=$ $\bigcup_{x>0}\left(\mathcal{A}^{\mu}\right)^{\prime}(x+\mathcal{E})$, where

$$
\left(\mathcal{A}^{\mu}\right)^{\prime}(x+\mathscr{E}) \triangleq\left\{c \in \mathcal{A}^{\mu}(x+\mathcal{E}): \sup _{\mathbb{Q} \in \mathcal{D}}\left(\mathbb{E} \int_{0}^{T} c(t) Y_{t}^{\mathbb{Q}} \mu(d t)-\left\langle\mathbb{Q}, \mathcal{E}_{T}\right\rangle\right)=x\right\} .
$$

Thus, by pointwise approximation of elements of $\bigcup_{x>0}\left(\mathcal{A}^{\mu}\right)^{\prime}(x+\mathscr{E})$ by elements of $\bigcup_{n \in \mathbb{N}} \wp_{n}$, we obtain

$$
\begin{aligned}
\lim _{n \rightarrow \infty} & \sup _{c \in S_{n}} \inf _{\mathbb{Q} \in \mathcal{D}}\left(\mathbb{E} \int_{0}^{T}\left(U(t, c(t))-y Y_{t}^{\mathbb{Q}} c(t)\right) \mu(d t)+y\left\langle\mathbb{Q}, \mathcal{E}_{T}\right\rangle\right) \\
= & \sup _{x>0} \sup _{c \in\left(\mathscr{A}^{\mu}\right)^{\prime}(x+\mathcal{E})} \mathbb{E}\left[\int_{0}^{T}(U(t, c(t))-x y) \mu(d t)\right]=\sup _{x>0}[\mathfrak{U}(x)-x y] .
\end{aligned}
$$

We define $V^{(n)}(t, y) \triangleq \sup _{0<x \leq n}[U(t, x)-x y]$, and the pointwise maximization yields

$$
\begin{aligned}
\inf _{\mathbb{Q} \in \mathcal{D}} & \sup _{c \in \delta_{n}}\left(\mathbb{E} \int_{0}^{T}\left(U(t, c(t))-y Y_{t}^{\mathbb{Q}} c(t)\right) \mu(d t)+y\left\langle\mathbb{Q}, \mathcal{E}_{T}\right\rangle\right) \\
= & \inf _{\mathbb{Q} \in \mathcal{D}}\left(\mathbb{E} \int_{0}^{T} V^{(n)}\left(t, y Y_{t}^{\mathbb{Q}}\right) \mu(d t)+y\left\langle\mathbb{Q}, \mathcal{E}_{T}\right\rangle\right) \triangleq \mathfrak{V}^{(n)}(y) .
\end{aligned}
$$

From (A.4)-(A.6) we conclude that $\lim _{n} \mathfrak{V}^{(n)}(y)=\sup _{x>0}[\mathfrak{U}(x)-x y]$. To prove the claim of the lemma it is enough to show that $\lim _{n \rightarrow \infty} \mathfrak{V}^{(n)}(y) \geq \mathfrak{V}(y)$, since $\mathfrak{V}^{(n)}(y) \leq \mathfrak{V}(y)$ holds for all $y>0, n \in \mathbb{N}$. For a fixed $y>0$, let $\left\{\overline{\mathbb{Q}}^{(n)}\right\}_{n \in \mathbb{N}} \subseteq \mathscr{D}$ be a sequence such that

$$
\lim _{n \rightarrow \infty}\left(\mathbb{E} \int_{0}^{T} V^{(n)}\left(t, y Y_{t}^{\mathbb{Q}^{(n)}}\right) \mu(d t)+y\left\langle\mathbb{Q}^{(n)}, \mathcal{E}_{T}\right\rangle\right)=\lim _{n \rightarrow \infty} \mathfrak{V}^{(n)}(y) .
$$

Using the construction from Lemma A.1 we can assume that $\left\langle\mathbb{Q}^{(n)}, \mathcal{E}_{T}\right\rangle \rightarrow$ $\left\langle\mathbb{Q}^{*}, \mathcal{E}_{T}\right\rangle$ and that $Y^{\mathbb{Q}^{(n)}} \rightarrow Y^{\mathbb{Q}^{*}}$ as $n \rightarrow \infty$, both in the $(\mu \otimes \mathbb{P})$-a.e. and in the Fatou sense, where $\mathbb{Q}^{*}$ is a cluster point of $\left\{\mathbb{Q}^{(n)}\right\}_{n \in \mathbb{N}}$. 
Let $\bar{U}(\cdot)$ be a majorant of $U$ and let $\bar{V}(\cdot)$ be its conjugate. Then it is easy to see that

$$
V^{(n)}(t, y) \leq \bar{V}^{(n)}(y):=\sup _{0<x \leq n}[\bar{U}(x)-x y] \quad \text { for all } t \in[0, T]
$$

and $\bar{V}^{(n)}(y)=\bar{V}(y)$ for $y \geq \bar{I}(1) \geq \bar{I}(n)$, where $\bar{I}(y):=\left(\bar{U}^{\prime}(\cdot)\right)^{-1}(y)$. The argument from Lemma A.1 takes care of the uniform integrability of the sequence of processes $\left\{V^{(n)}\left(\cdot, Y^{\mathbb{Q}^{(n)}}\right)^{-}\right\}_{n \in \mathbb{N}}$ as well as of the chain of inequalities

$$
\begin{aligned}
\lim _{n \rightarrow \infty} & \left(\mathbb{E} \int_{0}^{T} V^{(n)}\left(t, Y_{t}^{\mathbb{Q}^{(n)}}\right) \mu(d t)+y\left\langle\mathbb{Q}^{(n)}, \mathcal{E}_{T}\right\rangle\right) \\
\geq & \left(\mathbb{E} \int_{0}^{T} V\left(t, Y_{t}^{\mathbb{Q}^{*}}\right) \mu(d t)+y\left\langle\mathbb{Q}^{*}, \mathcal{E}_{T}\right\rangle\right) \geq \mathfrak{V}(y),
\end{aligned}
$$

settling the claim of the lemma.

REMARK 9. It is a consequence of the decrease of $\mathfrak{V}(\cdot)$ and the preservation of properness in the conjugacy relationship (see [32], Theorem 12.2, page 104) that Assumption 3.9 implies the existence of $y_{0}>0$ such that $\mathfrak{V}(y)<\infty$ for $y>y_{0}$. Furthermore, the strict convexity of $V(t, \cdot)$ allows us to denote by $\hat{\mathbb{Q}}^{y}$ the unique (as far as its action on $\varepsilon_{T}$ and the corresponding supermartingale $Y^{\hat{\mathbb{Q}}^{y}}$ are concerned) minimizer of the dual problem for $y$ such that $\mathfrak{V}(y)<\infty$.

LemmA A.4. $\mathfrak{V}(y) \in(-\infty, \infty)$ for all $y>0$.

Proof. Let $\underline{U}(\cdot)$ be a minorant of $U(\cdot, \cdot)$. Additionally, $\underline{U}(\cdot)$ is a utility function and the convex conjugate $\underline{V}(\cdot)$ of $\underline{U}(\cdot)$ satisfies $\underline{V}(y) \leq V(t, y)$ for all $t$. By the convexity of $\underline{V}(\cdot)$ and Jensen's inequality, we have

$$
\begin{aligned}
\mathfrak{V}(y) & =\inf _{\mathbb{Q} \in \mathcal{D}}\left(\mathbb{E}\left[\int_{0}^{T} V\left(t, y Y_{t}^{\mathbb{Q}}\right) \mu(d t)\right]+y\left\langle\mathbb{Q}, \mathcal{E}_{T}\right\rangle\right) \\
& \geq \inf _{\mathbb{Q} \in \mathcal{D}} \mathbb{E}\left[\int_{0}^{T} \underline{V}\left(y Y_{t}^{\mathbb{Q}}\right) \mu(d t)\right] \\
& \geq \inf _{\mathbb{Q} \in \mathcal{D}} \underline{V}\left(\mathbb{E}\left[\int_{0}^{T} y Y_{t}^{\mathbb{Q}} \mu(d t)\right]\right) \\
& \geq \underline{V}(y)>-\infty .
\end{aligned}
$$

To prove that $\mathfrak{V}(y)$ is finite, we first choose $y>0$ such that $\mathfrak{V}(y)<\infty$; its existence is guaranteed by Remark 9. For some $\gamma \in \Gamma_{3} \cap[\mathrm{AE}[U]$, 1) a.s. and some $0<\rho<1$, Proposition 3.7 implies that there exists $y_{0}>0$ such that

$$
V(t, \rho y) \leq C V(t, y) \quad \text { for } y \leq y_{0},
$$


where $C=\rho^{-\gamma /(1-\gamma)}$. By Proposition A.2 there is $\hat{\mathbb{Q}}^{y} \in \mathscr{D}$ such that $\mathfrak{V}(y)=$ $\mathbb{E}\left[\int_{0}^{T} V\left(t, y Y_{t}^{\hat{\mathbb{Q}}^{y}}\right) \mu(d t)\right]$, so

$$
\begin{aligned}
\mathfrak{V}(\rho y) \leq & \mathbb{E}\left[\int_{0}^{T} V\left(t, \rho y Y_{t}^{\hat{\mathbb{Q}}^{y}}\right) \mu(d t)\right] \\
= & \mathbb{E}\left[\int_{0}^{T} V\left(t, \rho y Y_{t}^{\hat{\mathbb{Q}}^{y}}\right) \mathbf{1}_{\left\{\rho y Y_{t}^{\hat{Q}^{y}}>y_{0}\right\}} \mu(d t)\right] \\
& +\mathbb{E}\left[\int_{0}^{T} V\left(t, \rho y Y_{t}^{\hat{\mathbb{Q}}^{y}}\right) \mathbf{1}_{\left\{\rho y Y_{t}^{\hat{\mathbb{Q}}^{y}} \leq y_{0}\right\}} \mu(d t)\right] \\
\leq & \sup _{t} V\left(t, y_{0}\right)+C \mathbb{E}\left[\int_{0}^{T} V\left(t, y Y_{t}^{\mathbb{Q}^{y}}\right) \mathbf{1}_{\left\{\rho y Y_{t}^{\hat{\mathbb{Q}}^{y}} \leq y_{0}\right\}} \mu(d t)\right]<\infty .
\end{aligned}
$$

We conclude that $\mathfrak{V}(y)<\infty$ for all $y>0$, due to the decrease of $\mathfrak{V}(\cdot)$.

Having established the existence and essential uniqueness of the solution, and the finiteness of the value function for the dual problem, we can apply ideas from the calculus of variations to obtain the following lemma:

Lemma A.5. For each $y>0$ and each $\mathbb{Q} \in \mathcal{D}$, we have

$$
\mathbb{E}\left[\int_{0}^{T}\left(Y_{t}^{\mathbb{Q}}-Y_{t}^{\hat{\mathbb{Q}}^{y}}\right) I\left(t, y Y_{t}^{\hat{\mathbb{Q}}^{y}}\right) \mu(d t)\right]+\left\langle\hat{\mathbb{Q}}^{y}-\mathbb{Q}, \mathcal{E}_{T}\right\rangle \leq 0,
$$

where $\hat{\mathbb{Q}}^{y}$ is the optimal solution to the dual problem of (3.3) (as in Proposition A.2 and Remark 9).

ProOF. For $y>0, \varepsilon \in(0,1)$ and $\mathbb{Q}^{\varepsilon}=(1-\varepsilon) \hat{\mathbb{Q}}^{y}+\varepsilon \mathbb{Q}$, the optimality of $\hat{\mathbb{Q}}^{y}$ implies

$$
\begin{aligned}
0 & \leq \mathbb{E}\left[\int_{0}^{T}\left(V\left(t, y Y_{t}^{\mathbb{Q}^{\varepsilon}}\right)-V\left(t, y Y_{t}^{\hat{\mathbb{Q}}^{y}}\right)\right) \mu(d t)\right]+y\left\langle\mathbb{Q}^{\varepsilon}-\hat{\mathbb{Q}}^{y}, \mathcal{E}_{T}\right\rangle \\
& \leq \mathbb{E}\left[\int_{0}^{T} y\left(Y_{t}^{\hat{\mathbb{Q}}^{y}}-Y_{t}^{\mathbb{Q}^{\varepsilon}}\right) I\left(t, y Y_{t}^{\mathbb{Q}^{\varepsilon}}\right) \mu(d t)\right]+y\left\langle\mathbb{Q}^{\varepsilon}-\hat{\mathbb{Q}}, \mathcal{E}_{T}\right\rangle \\
& =\varepsilon y\left(\mathbb{E}\left[\int_{0}^{T}\left(Y_{t}^{\hat{\mathbb{Q}}^{y}}-Y_{t}^{\mathbb{Q}}\right) I\left(t, y Y_{t}^{\mathbb{Q}^{\varepsilon}}\right) \mu(d t)\right]+\left\langle\hat{\mathbb{Q}}^{y}-\mathbb{Q}, \mathcal{E}_{t}\right\rangle\right) .
\end{aligned}
$$

Since

$$
\left(\left(Y_{t}^{\mathbb{Q}}-Y_{t}^{\hat{\mathbb{Q}}^{y}}\right) I\left(t, y Y_{t}^{\mathbb{Q}^{\varepsilon}}\right)\right)^{-} \leq Y_{t}^{\hat{\mathbb{Q}}^{y}} I\left(t, y Y_{t}^{\mathbb{Q}^{\varepsilon}}\right) \leq Y_{t}^{\hat{\mathbb{Q}}^{y}} I\left(t, y(1-\varepsilon) Y_{t}^{\hat{\mathbb{Q}}^{y}}\right),
$$

we can follow the same reasoning as in Lemma A.4 to show that the last term is dominated by a random process on $\Omega \times[0, T]$ which is $(\mu \otimes \mathbb{P})$-integrable. Now we can let $\varepsilon \rightarrow 0$ and apply Fatou's lemma to obtain the stated inequality. 
A.3. Differentiability of the value functions. We turn our attention to the differentiability properties of the value functions.

Proposition A.6. The dual value function $\mathfrak{V}(\cdot)$ is strictly convex and continuously differentiable on $\mathbb{R}_{+}$. Its derivative is given by

$$
\mathfrak{V}^{\prime}(y)=\left\langle\hat{\mathbb{Q}}^{y}, \mathcal{E}_{T}\right\rangle-\mathbb{E}\left[\int_{0}^{T} Y^{\hat{\mathbb{Q}}^{y}} I\left(t, y Y^{\hat{\mathbb{Q}}^{y}}\right) \mu(d t)\right] .
$$

PROOF. The fact that $\mathfrak{V}(\cdot)$ is strictly convex follows from the strict convexity of $V(t, \cdot)$. Therefore, to show that $\mathfrak{V}(\cdot)$ is continuously differentiable, it is enough (by convexity) to show that its derivative exists everywhere on $(0, \infty)$. We start by fixing $y>0$ and defining the function

$$
h(z) \triangleq \mathbb{E}\left[\int_{0}^{T} V\left(t, z Y_{t}^{\hat{\mathbb{Q}}^{y}}\right) \mu(d t)\right]+z\left\langle\hat{\mathbb{Q}}^{y}, \boldsymbol{E}_{T}\right\rangle, \quad z>0 .
$$

This function is convex and, by definition of the optimal solution $\hat{\mathbb{Q}}^{y}$ of the dual problem, we have $h(z) \geq \mathfrak{V}(z)$ for all $z>0$ and $h(y)=\mathfrak{V}(y)$. Again by convexity, we obtain

$$
\Delta^{-} h(y) \leq \Delta^{-} \mathfrak{V}(y) \leq \Delta^{+} \mathfrak{V}(y) \leq \Delta^{+} h(y),
$$

where $\Delta^{+}$and $\Delta^{-}$denote right and left derivatives, respectively. Now

$$
\begin{aligned}
\Delta^{+} h(y) & =\lim _{\varepsilon \rightarrow 0} \frac{h(y+\varepsilon)-h(y)}{\varepsilon} \\
& =\lim _{\varepsilon \rightarrow 0} \frac{1}{\varepsilon} \mathbb{E}\left[\int_{0}^{T} V\left(t,(y+\varepsilon) Y_{t}^{\hat{\mathbb{Q}}^{y}}\right)-V\left(t, y Y_{t}^{\hat{\mathbb{Q}}^{y}}\right) \mu(d t)\right]+\left\langle\hat{\mathbb{Q}}^{y}, \mathcal{E}_{T}\right\rangle \\
& \leq \liminf _{\varepsilon \rightarrow 0}\left(-\frac{1}{\varepsilon}\right) \mathbb{E}\left[\int_{0}^{T} \varepsilon Y_{t}^{\hat{\mathbb{Q}}^{y}} I\left(t,(y+\varepsilon) Y_{t}^{\hat{\mathbb{Q}}^{y}}\right) \mu(d t)\right]+\left\langle\hat{\mathbb{Q}}^{y}, \mathcal{E}_{T}\right\rangle \\
& =-\mathbb{E}\left[\int_{0}^{T} Y_{t}^{\hat{\mathbb{Q}}^{y}} I\left(t, y Y_{t}^{\hat{\mathbb{Q}}^{y}}\right) \mu(d t)\right]+\left\langle\hat{\mathbb{Q}}^{y}, \mathcal{E}_{T}\right\rangle
\end{aligned}
$$

by the monotone convergence theorem. Similarly, we get

$$
\Delta^{-} h(y) \geq \limsup _{\varepsilon \rightarrow 0} \mathbb{E}\left[-\int_{0}^{T} Y_{t}^{\hat{\mathbb{Q}}^{y}} I\left(t,(y-\varepsilon) Y_{t}^{\hat{\mathbb{Q}}^{y}}\right) \mu(d t)\right]+\left\langle\hat{\mathbb{Q}}^{y}, \mathcal{E}_{T}\right\rangle .
$$

Let $y_{0}$ be the constant from $\Gamma_{4}$, Lemma 3.7 , corresponding to some $\mathrm{AE}[U] \leq$ $\gamma<1$ a.s. Then

$$
\begin{aligned}
& \left|Y_{t}^{\hat{\mathbb{Q}}^{y}} I\left(t,(y-\varepsilon) Y_{t}^{\hat{\mathbb{Q}}^{y}}\right)\right| \\
& \quad \leq\left|Y_{t}^{\hat{\mathbb{Q}}^{y}} I\left(t,(y-\varepsilon) Y_{t}^{\hat{\mathbb{Q}}^{y}}\right)\right| \mathbf{1}_{\left\{Y_{t}^{\hat{\mathbb{Q}}^{y}} \leq y_{0} / y\right\}}+\left|Y_{t}^{\hat{\mathbb{Q}}^{y}} I\left(t,(y-\varepsilon) Y_{t}^{\hat{\mathbb{Q}}^{y}}\right)\right| \mathbf{1}_{\left\{Y_{t}^{\hat{\mathbb{Q}}^{y}}>y_{0} / y\right\}} .
\end{aligned}
$$


We fix $\varepsilon_{0}$ and observe that for $\varepsilon<\varepsilon_{0}$, by Lemma 3.7, the second part is dominated by

$$
\frac{1}{y-\varepsilon_{0}} \frac{\gamma}{1-\gamma} V\left(t,\left(y-\varepsilon_{0}\right) Y_{t}^{\hat{\mathbb{Q}}^{y}}\right) \leq \frac{1}{y-\varepsilon_{0}} \frac{\gamma}{1-\gamma} C V\left(t, y Y_{t}^{\hat{\mathbb{Q}}^{y}}\right),
$$

for some constant $C$. This last expression is in $L^{1}(\mu \otimes \mathbb{P})$, by finiteness of $\mathfrak{V}(\cdot)$. On the other hand, the first part in (A.8) is dominated by $K_{1}\left(\left(\left(y-\varepsilon_{0}\right) / y\right) y_{m}\right) Y_{t}^{\hat{\mathbb{Q}}^{y}}$, which is in $L^{1}(\mu \otimes \mathbb{P})$ by the supermartingale property of $Y^{\hat{\mathbb{Q}}^{y}}$. Having prepared the ground for the dominated convergence theorem, we can let $\varepsilon \rightarrow 0$ and obtain

$$
\Delta^{-} h(y) \geq\left\langle\hat{\mathbb{Q}}^{y}, \mathcal{E}_{T}\right\rangle-\mathbb{E}\left[\int_{0}^{T} Y_{t}^{\hat{\mathbb{Q}}^{y}} I\left(t, y Y_{t}^{\hat{\mathbb{Q}}^{y}}\right) \mu(d t)\right],
$$

completing the proof of the proposition.

LEMMA A.7. The dual value function $\mathfrak{V}(\cdot)$ has the following asymptotic behavior:

(i) $\mathfrak{V}^{\prime}(0+)=-\infty$.

(ii) $\mathfrak{V}^{\prime}(\infty) \in\left[\inf _{\mathbb{Q} \in \mathcal{D}}\left\langle\mathbb{Q}, \mathcal{E}_{T}\right\rangle, \sup _{\mathbb{Q} \in \mathscr{D}}\left\langle\mathbb{Q}, \mathcal{E}_{T}\right\rangle\right]$.

PROOF. (i) Suppose first there is a minorant $\underline{V}(\cdot)$ of $V(\cdot, \cdot)$ such that $\underline{V}(0+)=\infty$. Letting $y \rightarrow 0$ in (A.7), we get $\mathfrak{V}(0+)=\infty$ and, by convexity, $\mathfrak{V}^{\prime}(0+)=-\infty$. In the case when $\underline{V}(0+)<\infty$ for each minorant $\underline{V}(\cdot)$ of $V(\cdot, \cdot)$, we can easily construct a majorant $\overline{\bar{V}}(\cdot)$ such that $\bar{V}(0+)<\infty$, using the properties of functions $K_{1}$ and $K_{2}$ from Definition 3.1. We pick such a majorant $\bar{V}(\cdot)$, pick a minorant $\underline{V}(\cdot)$, set $\bar{I}(\cdot)=-\bar{V}^{\prime}(\cdot)$, set $D=\bar{V}(0+)-\underline{V}(0+)$ and choose $\mathbb{Q} \in \mathcal{D}$. Then, with $\rho=\left\|\mathscr{E}_{T}\right\|_{\mathbb{L}^{\infty}}$,

$$
\begin{aligned}
-\mathfrak{V}^{\prime}(y) & \geq \frac{\mathfrak{V}(0+)-\mathfrak{V}(y)}{y} \geq \frac{1}{y}[(\underline{V}(0+)-\bar{V}(0+))+\bar{V}(0+)-\mathfrak{V}(y)] \\
& \geq \frac{-D-\rho y}{y}+\frac{\bar{V}(0+)-\mathbb{E}\left[\int_{0}^{T} \bar{V}\left(y Y_{t}^{\mathbb{Q}}\right) \mu(d t)\right]}{y} \\
& \geq \frac{-D-\rho y}{y}+\mathbb{E}\left[\int_{0}^{T} Y_{t}^{\mathbb{Q}} \bar{I}\left(y Y_{t}^{\mathbb{Q}}\right) \mu(d t)\right] \rightarrow \infty \quad \text { as } y \downarrow 0,
\end{aligned}
$$

by the monotone convergence theorem.

(ii) By l'Hôpital's rule we have

$$
\begin{aligned}
\mathfrak{V}^{\prime}(\infty) & =\lim _{y \rightarrow \infty} \frac{\mathfrak{V}(y)}{y}=\lim _{y \rightarrow \infty} \frac{\inf _{\mathbb{Q} \in \mathcal{D}}\left(\mathbb{E}\left[\int_{0}^{T} V\left(t, y Y_{t}^{\mathbb{Q}}\right) \mu(d t)\right]+y\left\langle\mathbb{Q}, \mathcal{E}_{T}\right\rangle\right)}{y} \\
& \in\left[L+\inf _{\mathbb{Q} \in \mathcal{D}}\left\langle\mathbb{Q}, \mathcal{E}_{T}\right\rangle, L+\sup _{\mathbb{Q} \in \mathscr{D}}\left\langle\mathbb{Q}, \mathcal{E}_{T}\right\rangle\right],
\end{aligned}
$$


where $L \triangleq \lim _{y \rightarrow \infty} \frac{1}{y} \inf _{\mathbb{Q} \in \mathcal{D}} \mathbb{E}\left[\int_{0}^{T} V\left(t, y Y_{t}^{\mathbb{Q}}\right) \mu(d t)\right]$. From the Definition 3.1 of the utility function, we read $\partial_{2} V(t, y) \leq-\left(K_{1}\right)^{-1}(y) \rightarrow 0$ when $y \rightarrow \infty$, so for an $\varepsilon>0$ we can find a constant $C(\varepsilon)$ such that $-V(t, y) \leq C(\varepsilon)+\varepsilon y$ for all $t \in[0, T]$ and all $y>0$. To complete the proof, we denote by $\mathfrak{V}_{0}(\cdot)$ the (strictly convex, decreasing) value function of the dual optimization problem (3.3) when $\mathcal{E}_{T} \equiv 0$. Then the decrease of $\mathfrak{V}_{0}(\cdot)$ and l'Hôpital's rule imply

$$
\begin{aligned}
0 & \leq-\mathfrak{V}_{0}^{\prime}(\infty)=\lim _{y \rightarrow \infty} \frac{-\mathfrak{V}_{0}(y)}{y}=\lim _{y \rightarrow \infty} \sup _{\mathbb{Q} \in \mathcal{D}} \frac{1}{y} \mathbb{E}\left[\int_{0}^{T}-V\left(t, y Y_{t}^{\mathbb{Q}}\right) \mu(d t)\right]=-L \\
& \leq \lim _{y \rightarrow \infty} \sup _{\mathbb{Q} \in \mathscr{D}} \frac{1}{y} \mathbb{E}\left[\int_{0}^{T}\left(C(\varepsilon)+\varepsilon y Y_{t}^{\mathbb{Q}}\right) \mu(d t)\right] \\
& \leq \lim _{y \rightarrow \infty} \mathbb{E} \int_{0}^{T}\left(\frac{C(\varepsilon)}{y}+\varepsilon\right) \mu(d t)=\varepsilon .
\end{aligned}
$$

Consequently, $L=0$ and the claim follows.

A.4. Proof of the main result, Theorem 3.10. In this section we combine the preceding lemmata and propositions to complete the proof of Theorem 3.10.

(i) By the concavity of $U(t, \cdot)$ and Assumption 3.9, we deduce that $\mathfrak{U}(x)<\infty$ for any $x>0$. For $x>0$ we define $c(t) \triangleq x \forall t \in[0, T]$. Then $c \in \mathcal{A}^{\mu}(x+\mathcal{E})$, because the constant consumption-rate process $c(\cdot) \equiv x$ can be financed by the trivial portfolio $H \equiv 0$ and initial wealth only. Since

$$
\begin{aligned}
\mathfrak{U}(x) & \geq \mathbb{E}\left[\int_{0}^{T} U(t, c(t)) \mu(d t)\right]=\mathbb{E}\left[\int_{0}^{T} U(t, x) \mu(d t)\right] \\
& \geq \mathbb{E}\left[\int_{0}^{T} \underline{U}(x) \mu(d t)\right]=\underline{U}(x)>-\infty,
\end{aligned}
$$

we conclude that $|\mathfrak{U}(x)|<\infty$ for all $x>0$. The assertion that $|\mathfrak{V}(y)|<\infty$ for all $y>0$ is the content of Lemma A.4.

(ii) $\mathfrak{V}(\cdot)$ is continuously differentiable by Proposition A.6. From the conjugacy relationship in Lemma A.3 and the properties of convex conjugation (see Theorem 26.5 in [32]), we deduce the continuous differentiability of $\mathfrak{U}(\cdot)$.

(iii) Follows from Lemma A.3 and the properties of convex conjugation (see Theorem 12.2 in [32]).

(iv) The assertion is a direct consequence of Lemma A.6 and the properties of convex conjugation (see Theorem 26.5 in [32]).

(vi) Follows from Lemma A.6.

(v) The dual problem has an essentially unique solution $\hat{\mathbb{Q}}^{y} \in \mathscr{D}$ for any $y>0$, by Proposition A.2 and Remark 9. To establish the result for the primal problem, we pick $x>0$, a solution $\hat{\mathbb{Q}}^{y}$ of the dual problem corresponding to 
$y=\mathfrak{U}^{\prime}(x)$, and define $\hat{c}^{x}(t) \triangleq I\left(t, y Y_{t}^{\hat{\mathbb{Q}}^{y}}\right)$ for all $t \in[0, T]$. Then the relationship $-\mathfrak{V}^{\prime}(y)=\left(\mathfrak{U}^{\prime}(\cdot)\right)^{-1}(y), y>0$ (see [32], Theorem 26.6), and Proposition A.6 give

$$
\mathbb{E}\left[\int_{0}^{T} \hat{c}^{x}(t) Y_{t}^{\hat{\mathbb{Q}}^{y}} \mu(d t)\right]=-\mathfrak{V}^{\prime}(y)+\left\langle\hat{\mathbb{Q}}^{y}, \mathcal{E}_{T}\right\rangle=x+\left\langle\hat{\mathbb{Q}}^{y}, \mathcal{E}_{T}\right\rangle,
$$

so for any $\mathbb{Q} \in \mathscr{D}$, by Proposition A.5,

$$
\begin{aligned}
& \mathbb{E}\left[\int_{0}^{T} \hat{c}^{x}(t) Y_{t}^{\mathbb{Q}} \mu(d t)\right] \\
& \quad \leq \mathbb{E}\left[\int_{0}^{T} \hat{c}^{x}(t) Y_{t}^{\hat{\mathbb{Q}}^{y}} \mu(d t)\right]+\left\langle\mathbb{Q}, \mathcal{E}_{T}\right\rangle-\left\langle\hat{\mathbb{Q}}^{y}, \mathcal{E}_{T}\right\rangle=x+\left\langle\mathbb{Q}, \mathcal{E}_{T}\right\rangle .
\end{aligned}
$$

Thus $\hat{c}^{x}(\cdot) \in \mathcal{A}(x+\mathcal{E})$ by the characterization of admissible consumption processes in Proposition 2.13.

Having established the admissibility of $\hat{c}^{x}(\cdot)$, we note that

$$
\begin{aligned}
\mathbb{E}\left[\int_{0}^{T} U\left(t, \hat{c}^{x}(t)\right) \mu(d t)\right] \\
\quad=\mathbb{E}\left[\int_{0}^{T} V\left(t, y Y_{t}^{\hat{\mathbb{Q}}^{y}}\right) \mu(d t)\right]+\mathbb{E}\left[\int_{0}^{T} y Y_{t}^{\hat{\mathbb{Q}}^{y}} I\left(t, y Y_{t}^{\hat{\mathbb{Q}}^{y}}\right) \mu(d t)\right] \\
\quad=\mathfrak{V}(y)-y \mathfrak{V}^{\prime}(y)=\mathfrak{U}(x),
\end{aligned}
$$

by the conjugacy relation (iii), the expression for the derivative of the dual value function (vi) and the definition of $y$. This closes the duality gap and proves the optimality of $\hat{c}^{x}(\cdot)$.

\section{REFERENCES}

[1] Bhaskara RaO, K. P. S. and Bhaskara Rao, M. (1983). Theory of Charges. Academic Press, London.

[2] Brannath, W. and Schachermayer, W. (1999). A bipolar theorem for subsets of $L_{+}^{0}(\Omega, \mathcal{F}, \mathcal{P})$. Séminaire de Probabilités XXXIII. Lecture Notes in Math. 1709 349-354. Springer, Berlin.

[3] Chung, K.-L. (1974). A Course in Probability Theory, 2nd ed. Academic Press, New York.

[4] CoX, J. C. and HuANG, C. F. (1989). Optimal consumption and portfolio policies when asset prices follow a diffusion process. J. Econom. Theory $4933-83$.

[5] Cox, J. C. and HuAng, C. F. (1991). A variational problem arising in financial economics. J. Math. Econom. 20 465-487.

[6] CuOCO, D. (1997). Optimal consumption and equilibrium prices with portfolio constraints and stochastic income. J. Econom. Theory 72 33-73.

[7] CVITANić, J. and KARATZAS, I. (1992). Convex duality in constrained portfolio optimization. Ann. Appl. Probab. 2 767-818.

[8] Cvitanić, J., Schachermayer, W. and Wang, H. (2001). Utility maximization in incomplete markets with random endowment. Finance Stoch. 5 237-259. 
[9] Delbaen, F. and Schachermayer, W. (1993). A general version of the fundamental theorem of asset pricing. Math. Ann. 300 463-520.

[10] Delbaen, F. and Schachermayer, W. (1995). The existence of absolutely continuous local martingale measures. Ann. Appl. Probab. 5 926-945.

[11] Delbaen, F. and Schachermayer, W. (1998). The fundamental theorem of asset pricing for unbounded stochastic processes. Math. Ann. 312 215-250.

[12] Delbaen, F. and Schachermayer, W. (1999). A compactness principle for bounded sequences of martingales with applications. In Proceedings of the Seminar on Stochastic Analysis, Random Fields and Applications 137-173. Birkhäuser, Basel.

[13] Dellacherie, C. and Meyer, P.-A. (1982). Probabilities and Potential, Vol. B: Theory of Martingales. North-Holland, Amsterdam.

[14] El Karoui, N. and JeanblanC-Picqué, M. (1998). Optimization of consumption with labor income. Finance Stoch. 2 409-440.

[15] El Karoui, N. and QueneZ, M.-C. (1995). Dynamic programming and pricing of contingent claims in an incomplete market. SIAM J. Control Optim. 33 29-66.

[16] Föllmer, H. and Kramkov, D. (1997). Optional decomposition under constraints. Probab. Theory Related Fields 109 1-25.

[17] He, H. and Pearson, N. D. (1991). Consumption and portfolio policies with incomplete markets and short-sale constraints: The finite-dimensional case. Math. Finance 1 1-10.

[18] He, H. and Pearson, N. D. (1991). Consumption and portfolio policies with incomplete markets and short-sale constraints: The infinite-dimensional case. J. Econom. Theory $\mathbf{5 4}$ 259-304.

[19] JACOD, J. (1979). Calcul stochastique et problèmes de martingales. Lecture Notes in Math. 714. Springer, Berlin.

[20] Karatzas, I. and Shreve, S. E. (1991). Brownian Motion and Stochastic Calculus, 2 nd ed. Springer, New York.

[21] Karatzas, I. and Shreve, S. E. (1998). Methods of Mathematical Finance. Springer, New York.

[22] Karatzas, I., Lehoczky, J. P. and Shreve, S. E. (1987). Optimal portfolio and consumption decisions for a "small investor" on a finite horizon. SIAM J. Control Optim. 25 1557-1586.

[23] Karatzas, I., Lehoczky, J. P., Shreve, S. E. and XU, G. L. (1991). Martingale and duality methods for utility maximization in an incomplete market. SIAM J. Control Optim. 29 702-730.

[24] Komlós, J. (1967). A generalization of a problem of Steinhaus. Acta Math. Hungar. 18 217-229.

[25] KRAmKOV, D. (1996). Optional decomposition of supermartingales and hedging contingent claims in incomplete security markets. Probab. Theory Related Fields 105 459-479.

[26] Kramkov, D. and Schachermayer, W. (1999). A condition on the asymptotic elasticity of utility functions and optimal investment in incomplete markets. Ann. Appl. Probab. 9 904-950.

[27] LAKNer, P. and Slud, E. (1991). Optimal consumption by a bond investor: The case of random interest rate adapted to a point process. SIAM J. Control Optim. 29 638-655.

[28] Merton, R. C. (1969). Lifetime portfolio selection under uncertainty: The continuous-time case. Review of Economic Statistics $51247-257$.

[29] Merton, R. C. (1971). Optimum consumption and portfolio rules in a conitinuous-time model. J. Econom. Theory 3 373-413.

[30] PliskA, S. R. (1986). A stochastic calculus model of continuous trading: Optimal portfolio. Math. Oper. Res. 11 371-382.

[31] Protter, PH. (1990). Stochastic Integration and Differential Equations. Springer, New York. 
[32] Rockafellar, R. T. (1970). Convex Analysis. Princeton Univ. Press.

[33] SChachermayer, W. (2000). Optimal investment in incomplete financial markets. In Proceedings of the First World Congress of the Bachelier Society 427-462. Springer, Berlin.

[34] SchwartZ, M. (1986). New proofs of a theorem of Komlós. Acta Math. Hungar. 47 181-185.

[35] Shiryaev, A. N. (1996). Probability, 2nd ed. Springer, New York. [Translated from the first (1980) Russian edition by R. P. Boas.]

[36] Strasser, H. (1985). Mathematical Theory of Statistics. de Gruyter, Berlin.

[37] WojatszCYK, P. (1996). Banach Spaces for Analysts. Cambridge Univ. Press.

[38] XU, G.-L. (1990). A duality method for optimal consumption and investment under shortselling prohibition. Ph.D. dissertation, Dept. Mathematics, Carnegie-Mellon Univ.

[39] Yosida, K. and HewitT, E. (1952). Finitely additive measures. Trans. Amer. Math. Soc. 72 46-66.

[40] Žıtković, G. (2002). A filtered version of the bipolar theorem of Brannath and Schachermayer. J. Theoret. Probab. 15 41-61.

DEPARTMENTS OF MATHEMATICS AND STATISTICS

COLUMBIA UNIVERSITY

NEW YORK, NEW YORK 10027

E-MAIL: ik@math.columbia.edu
DEPARTMENT OF STATISTICS

COLUMBIA UNIVERSITY

NEW York, NEW York 10027

E-MAIL: gordanz@stat.columbia.edu 